\title{
TV/Series
}

14 | 2018

Posthumains en séries

\section{Le sexe sans le sexe : convergence de la nudité frontale et de l'empowerment féminin dans Westworld}

Benjamin Campion

\section{OpenEdition}

Journals

Édition électronique

URL : http://journals.openedition.org/tvseries/2856

DOI : $10.4000 /$ tvseries.2856

ISSN : 2266-0909

Éditeur

GRIC - Groupe de recherche Identités et Cultures

Référence électronique

Benjamin Campion, «Le sexe sans le sexe : convergence de la nudité frontale et de l'empowerment féminin dans Westworld", TV/Series [En ligne], 14 | 2018, mis en ligne le 31 décembre 2018, consulté le 19 avril 2019. URL : http://journals.openedition.org/tvseries/2856 ; DOI : 10.4000/tvseries.2856

Ce document a été généré automatiquement le 19 avril 2019

\section{(c) (1) () $\Theta$}

$T V /$ Series est mis à disposition selon les termes de la licence Creative Commons Attribution - Pas d'Utilisation Commerciale - Pas de Modification 4.0 International. 


\title{
Le sexe sans le sexe : convergence de la nudité frontale et de l' empowerment féminin dans Westworld
}

\author{
Benjamin Campion
}

1 «Il ne voyait qu'une jolie silhouette, de jolies jambes... mais d'une manière indéfinissable son allure avait quelque chose d'érotique ${ }^{1}$. »

2 Commençons par des mots qui se voulurent définitifs : «Je sais quand j'en ai sous les yeux 2.» Si cette affirmation du juge américain Potter Stewart, se prononçant là sur le caractère (en l'occurrence non-) pornographique des Amants de Louis Malle (1958), est tant restée dans les mémoires, c'est non seulement parce qu'elle continue aujourd'hui de faire jurisprudence aux États-Unis, mais aussi parce qu'elle a très tôt positionné le débat de l'obscénité cinématographique - et, par extension, télévisuelle - sur le plan de l'image. Voir, c'est savoir : en suivant cette logique proverbiale, un gros plan de pénis en érection renverrait automatiquement le diffuseur à l'obscénité par son adoption d'une convention formelle du cinéma pornographique. Et même si Stewart prit le soin d'assumer la totale subjectivité de son propos et de consentir que le degré d'offense pût varier d'un spectateur à l'autre selon son niveau de sensibilité, son raisonnement en apparence frappé au coin du bon sens nous amène de manière ambiguë à considérer que la seule vision d'images à caractère pornographique suffirait à nous renseigner sur leur facture délictueuse.

Or, l'impression que renvoie la première saison de la série américaine Westworld (HBO, 2016-) - que je nommerai ici «Westworld» tout court, en ayant bien conscience que l'analyse d'une série en cours de production sera toujours susceptible d'être démentie par ses développements ultérieurs - est tout sauf celle de la pornographie. Entre jeux d'évitement partiel (par l'obscurité, le resserrement du cadre, l'éloignement de la caméra, l'intégration de caches au décor) et brefs plans d'exposition frontale, la série n'hésite certes pas à nous livrer ses corps dans le plus simple appareil. Elle ne louvoie pas non plus 
au moment d'assumer la tonalité et l'esthétique érotiques (voire pornographiques soft, c'est-à-dire sans monstration de pénétrations ni de pénis en érection) des virages pris par son récit, comme nous le verrons dans la suite de cet article. Mais l'accuser de montrer trop fréquemment la «chair fraîche" afin d'attirer le spectateur à moindre frais scénaristique reviendrait à lui intenter le type de procès d'intention dont, pour continuer d'exister, l'art subversif a plus que jamais besoin de se voir scrupuleusement préservé.

D'autant qu'en l'occurrence, la latitude offerte par le positionnement de la série sur le câble premium américain s'est avant tout pliée à la vision globale de ses auteurs sur la dimension psychanalytique $\mathrm{du}$ monde westworldien, labyrinthe intérieur dont la pénétration symbolique (et pourtant littérale) du «corps» destine le plus avisé des explorateurs à atteindre l'âme. Certains y liront une crainte de la part des showrunners de la série - le couple Jonathan Nolan-Lisa Joy - de soulever les mêmes polémiques que Game of Thrones (HBO, 2011-), prédécesseur à succès souvent brocardé pour la prétendue « gratuité » de ses scènes de sexe (si tant est qu'un tel substantif puisse être employé, non accompagné d'une quelconque définition, dans le cadre d'une critique ou d'une analyse filmique) $)^{3}$. Je postule quant à moi l'idée d'une tentative de conciliation entre nudité frontale et télévision américaine non spécialisée, en dépit d'une cohabitation souvent tumultueuse par le passé. Car si, pour reprendre l'expression de Linda Williams, le cinéma hollywoodien n'est jamais véritablement sorti de sa « longue adolescence ${ }^{4} »-$ exception faite des parenthèses désinhibées que furent le pré-code (1930-34) et le Nouvel Hollywood (fin 1960-début 1980) -, il m'apparaît a contrario que HBO poursuit avec Westworld sa formulation d'une télévision " adulte » abordant la nudité non comme un sujet à risques, mais comme l'une des composantes naturelles d'une expression quotidienne n'appelant pas à la fausse pudibonderie. La chaîne poursuit ainsi une réflexion initiée neuf ans plus tôt par l'entremise de Tell Me You Love Me (2007), en déplaçant le curseur du sexe explicite - infranchissable Rubicon de la fiction télévisuelle américaine? - vers la nudité frontale, tout en adoptant certains codes du blockbuster plutôt que du cinéma indépendant de niche 5 .

5 À ce titre, il est à noter que Westworld (adaptation d'un film éponyme de 1973 écrit et réalisé par Michael Crichton) a vu le jour au moment même où la science-fiction hollywoodienne commençait à perdre ostensiblement de sa force émotionnelle et de sa propension à incarner le trouble érotique. En témoigne ce constat désabusé de JeanSébastien Chauvin, s'exprimant là au moment de dresser le bilan de l'année cinématographique 2017: «La fascination pour les robots (ce que sont aussi les superhéros) a fait basculer l'imaginaire hollywoodien dans un monde inorganique et sans vie ${ }^{6}$ .» À l'inverse, les robots (ou "hôtes ») de Westworld sont tout sauf des machines infaillibles et surpuissantes dont le corps sans aspérités se réduirait à un simple accessoire leur permettant de prendre systématiquement l'ascendant sur leurs adversaires. Ce sont des êtres sensibles, faits de chair et de sang, qui pleurent leurs proches disparus, endurent mille morts et ne se recomposent que par la grâce du bistouri humain et de l'effacement de leur mémoire à court, à moyen et (sauf dysfonctionnement...) à long terme.

6 Mon hypothèse est que cette conscience corporelle - charnelle, pourrait-on dire - du posthumain westworldien sert paradoxalement une érotique de l'intériorité plutôt que de l'effeuillage ou de l'accouplement traditionnel. Les corps sont déjà nus au moment où débute la série, ce qui s'apparente à une manière d'évacuer toute gêne et tout suspense inhérents à la révélation de ce qui se cache «là-dessous » (à la manière d'un Alain 
Guiraudie ouvrant son film L'Inconnu du lac par un plan "à hauteur de couilles ${ }^{7}$ » - les siennes, en l'occurrence - afin de mettre d'emblée ses acteurs et son équipe de tournage à l'aise et d'entrer sans circonvolutions dans le vif du sujet). Westworld met ainsi la corporéité affichée sans fausse pudeur de ses hôtes brimés et exploités par l'homme au service de ficelles classiques (mais toujours aussi irrésistibles) d'empowerment féminin, selon cette dialectique "extrême centriste" qui consiste pour HBO, depuis son positionnement sur le créneau des séries "non télévisuelles » en 1996, à ne pas se contenter de reproduire des formules toutes faites sans chercher à les égratigner et à les métisser. En l'absence de traduction satisfaisante, j'emploierai ici le mot empowerment en langue originale, tel que l'ont défini Marie-Hélène Bacqué et Carole Biewener :

7 L'empowerment articule deux dimensions, celle du pouvoir, qui constitue la racine du mot, et celle du processus d'apprentissage pour y accéder. Il peut désigner autant un état (être empowered) qu'un processus, cet état et ce processus étant à la fois individuels, collectifs et sociaux ou politiques - même si, selon les usages de la notion, l'accent est mis sur l'une de ces dimensions ou au contraire sur leur articulation. Cela implique une démarche d'autoréalisation et d'émancipation des individus, de reconnaissance des groupes ou des communautés et de transformation sociale ${ }^{8}$.

8 Je distinguerai également narration et attraction en marchant dans les pas de Tom Gunning, lequel s'est fondé sur les préceptes suivants pour poser les bases de son archéologie du cinéma :

Qu'est-ce qu'au juste le cinéma d'attraction? Il s'agit en premier lieu d'un cinéma fondé sur la qualité célébrée par Léger, la capacité à montrer quelque chose. Par contraste avec la dimension voyeuriste du cinéma narratif analysée par Christian Metz, ce cinéma serait plutôt exhibitionniste. [...] Du grimacement des comédiens vers la caméra aux révérences et aux gesticulations constantes des prestidigitateurs dans les films de magie, ce cinéma étale sa visibilité et accepte de sacrifier l'apparente autonomie de l'univers de la fiction si cela lui permet de solliciter l'attention du spectateur ${ }^{9}$

9 Pour étayer ma démonstration, je m'appuierai en outre sur deux scènes (littéralement) centrales de la première saison, qui font office de pivots pour des hôtesses ${ }^{10}$ bien résolues à enfin jouir de leur propre liberté d'agir et de penser en sortant de la boucle narrative dans laquelle les a enfermées le scénario de Westworld (le parc et, à travers lui, la série). La première scène se déroule à Pariah, repaire de brigands et de mercenaires réputé pour ses bordels aux mille délices interlopes (Contrapasso, S01E05). Lors d'une orgie filmée selon les codes esthétiques du softporn, Dolores Abernathy, la plus ancienne hôtesse de Westworld, découvre sa véritable nature et parvient (pour un temps) à prendre son destin en main. Si la représentation sexuelle sert de toile de fond à cette épiphanie, c'est néanmoins l'appropriation d'un nouveau costume qui permet à Dolores de faire ressurgir son moi antérieur. La seconde scène sur laquelle je souhaite m'attarder montre Felix Lutz, technicien de laboratoire s'attelant d'ordinaire à remettre les hôtes endommagés sur pied, en train de procurer un plaisir inédit à Maeve Millay (autre hôtesse de première génération sur la voie de l'affranchissement) en poussant ses capacités intellectuelles audelà de la limite autorisée (The Adversary, S01E06). Là encore, nous constaterons que la jouissance ne passe pas par la fusion charnelle souvent fantasmée de l'homme et de la machine - fusion présentée tout au long de la série comme un acte pervers, malsain et obscène -, mais par l'optimisation d'un personnage de fiction s'arrogeant de lui-même de 
nouveaux attributs. Je conclurai cet article en arguant que l'omniprésence de la nudité frontale (in ou hors-champ) permet à Westworld de repousser les limites de la représentation sexuelle, non pas en occultant celle-ci - ce qui reviendrait à fuir devant la difficulté -, mais en détournant son régime attractionnel primaire pour mieux s'inscrire dans la durée. Car, contrairement à un film pornographique, la série ne considère pas le sexe comme une finalité, mais comme le début d'une longue histoire.

\section{De la demoiselle en détresse à l'intrépide justicière : la mue érotique de Dolores}

Sans le savoir, Dolores revit inéluctablement la même journée. Elle est l'une des attractions principales d'un parc aux allures de Far West: Westworld, exutoire grandeur nature assurant à de riches visiteurs (ou "guests", en version originale) de pouvoir assouvir leurs fantasmes les plus délurés de whisky on the rocks, de sexe dépravé et de duels au soleil se pratiquant en toute impunité (Fig. 1).

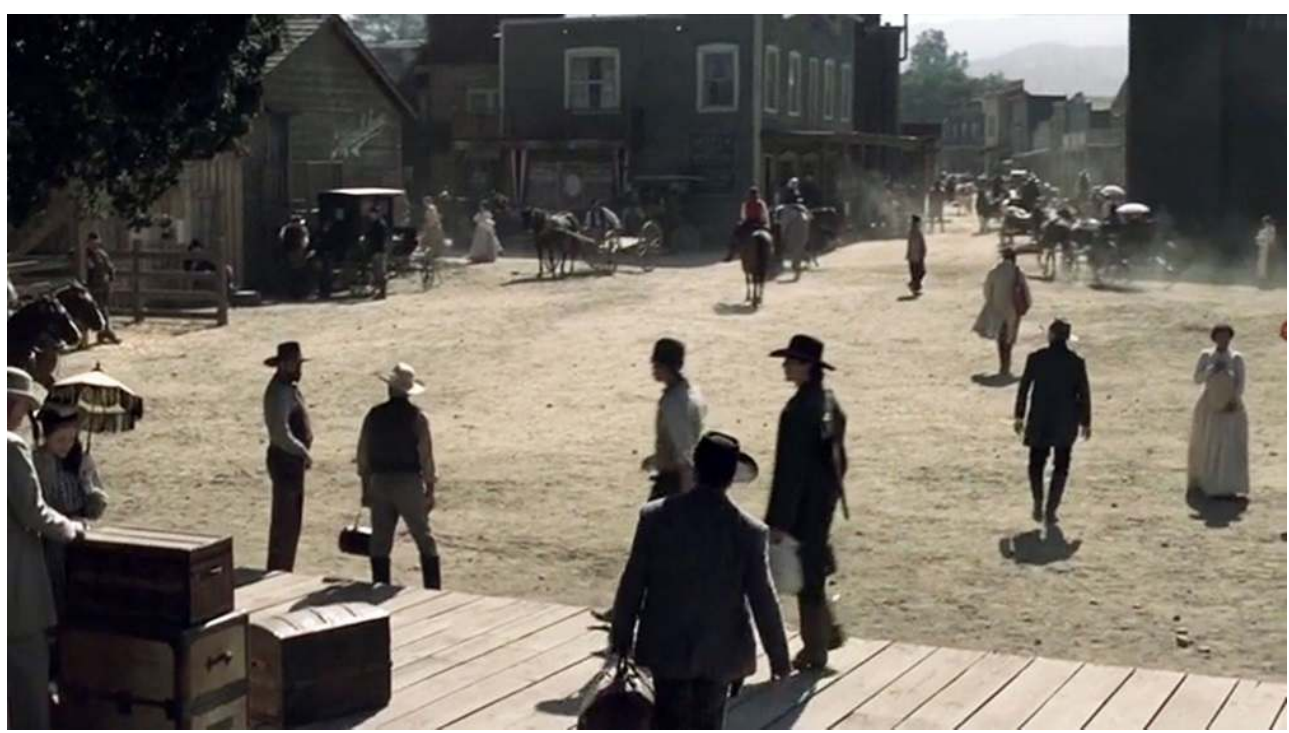

Fig. 1 : L'arrivée de Teddy Flood dans le parc (S01E01).

11 Le réalisme du parc a toutefois ses limites : si les visiteurs peuvent interagir avec les hôtes au point de les pousser à une improvisation partielle, ces derniers n'ont d'autre choix que de rejouer chaque jour le même scénario prédéfini, en attendant que leurs auteurs (humains) se décident à réorienter leur sort. Et si les visiteurs peuvent infliger aux hôtes les pires sévices corporels, ces derniers se voient dans l'incapacité de répliquer, dans la mesure où leurs coups ne portent pas et où leurs balles restent sans effet sur les humains. Précisons en outre que, quand ils meurent, les hôtes sont envoyés en réfection et leur mémoire réinitialisée afin de leur éviter le traumatisme des souffrances passées. Acteurs malgré eux d'une vie qu'ils croient contrôler, ces "pantins " peuvent également se voir affecter un autre rôle ou être renvoyés en réserve, en attendant que le showrunner leur trouve une nouvelle utilité (Fig. 2). En surplomb du parc, c'est même toute une équipe artistique qui veille au bon fonctionnement du " plateau de tournage », du scénariste en chef aux directeurs d'acteurs, des décorateurs aux costumiers, des illustrateurs aux maquilleurs (Fig. 3). 


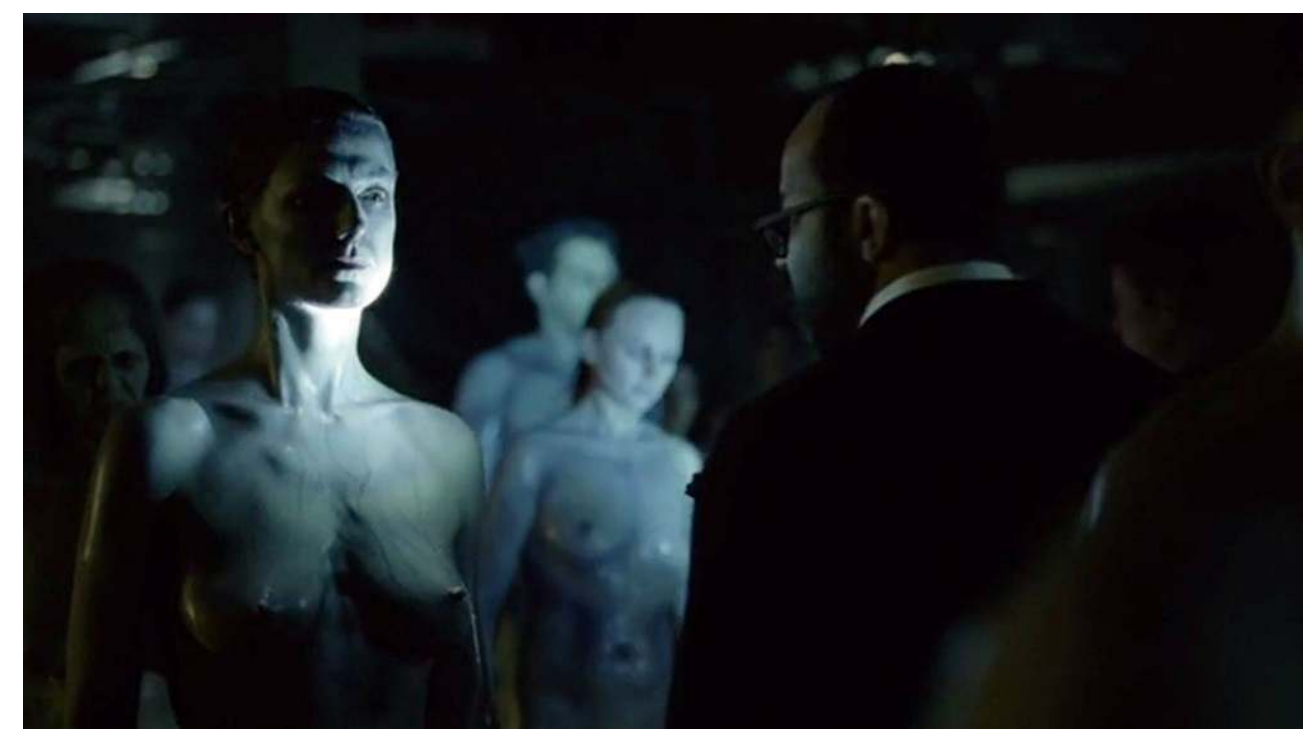

Fig. 2 : Visite de la réserve d'hôtes désactivés (S01E01).

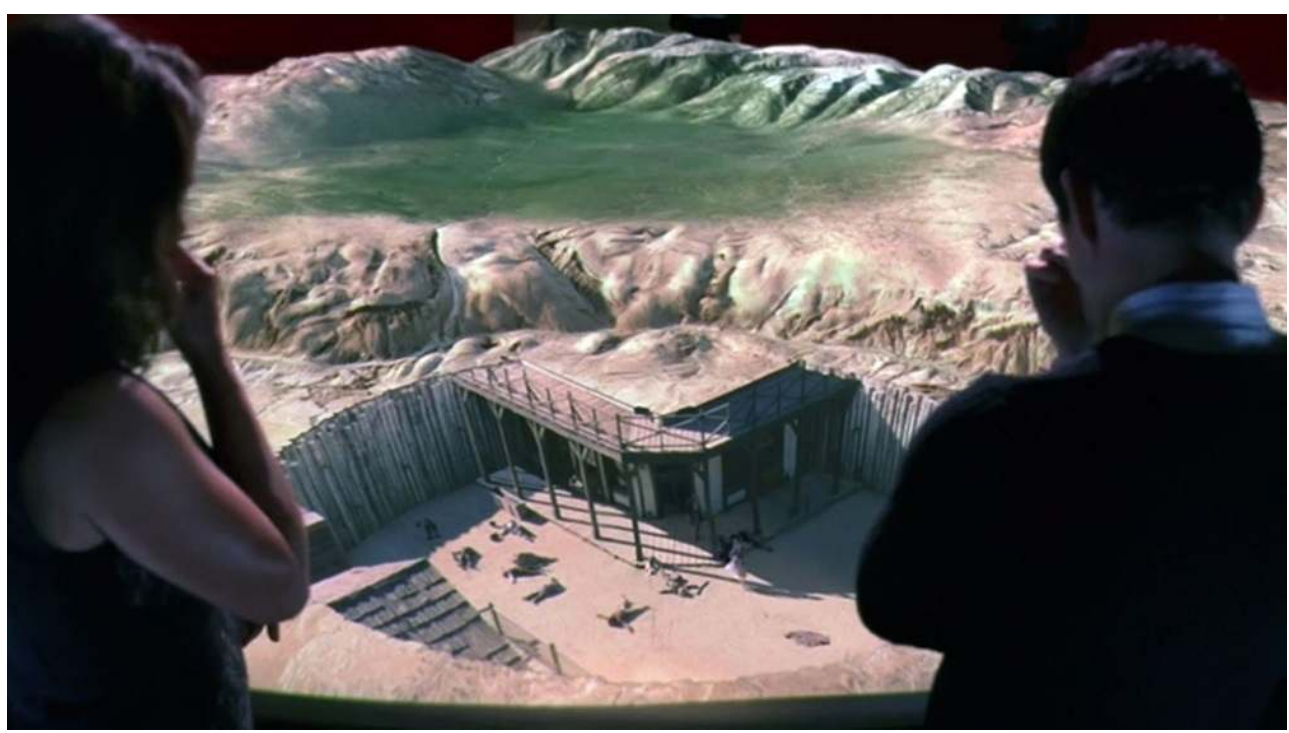

Fig. 3 : Vue du parc miniaturisé en surplomb (S01E01).

Dolores est donc une hôtesse au double sens du terme : elle habite un corps spécialement conçu et confectionné pour elle, et elle livre celui-ci en pâture à des visiteurs libres de le manipuler et de le maltraiter comme bon leur semble. Si la série reprend à son compte des recettes déjà bien éprouvées du récit littéraire et cinématographique de sciencefiction - l'exploitation de la machine par l'homme, la dérive assumée du "doux rêveur " dans un monde qu'il a lui-même imaginé, la rébellion de la créature contre son géniteur mégalomane - l'affirmation de son identité passe donc avant tout par une interrogation du corps et de la nature de cet être à qui l'on impose de vivre au présent pour ne pas (trop) le voir dévier de sa trajectoire initiale à force de ressasser le passé. Le récit global a beau s'enclencher, comme le préconise tout manuel de scénario, par un dérèglement inattendu amenant certains "élus » à sortir de leurs rails d'acteurs malgré eux (on apprend rapidement qu'une mise à jour logicielle leur ouvre les portes de la rêverie, synonyme de réminiscence et de soif de justice), ces derniers continuent dans un premier temps de subir les assauts répétés de l'humain dans ce qu'il a de plus bestial et de plus pernicieux. 


\section{Poupée de première génération}

À ce titre, il n'est pas anodin que Dolores possède un physique évoquant (tout en le renvoyant à des mensurations paradoxalement plus humaines) celui d'une poupée Barbie : une silhouette longiligne, un corps svelte aux courbes discrètes, un port altier, une longue chevelure blonde, un visage de porcelaine, des yeux bleus qui semblent ne jamais devoir ciller (même quand une mouche les arpente impudemment de ses pattes fureteuses, comme dans la scène d'ouverture de la série). La longue robe d'un bleu " virginal ${ }^{11}$ » qu'elle porte tout au long de la première moitié de la saison s'apparente d'ailleurs à l'unique accessoire que trouvera l'enfant dans la boîte du modèle « Dolores, fille d'éleveur » commandée à Noël (Fig. 4). Logan, habitué du parc cherchant à désinhiber son beau-frère William en lui offrant une première visite qu'il espère inoubliable, emploie d'ailleurs lui-même le mot " poupée $^{12}$ » (S01E05) pour désigner cette hôtesse candide et conciliante en toute circonstance dont il se moque bien de savoir si elle va goûter ou non les effluves lascifs et libidineux de l'une de ces orgies tribales dont Pariah a le secret. Très vite, Westworld pose ainsi les principes esthétiques et moraux d'une plastique figurale mettant l'accent sur la malléabilité et l'exploitation de corps féminins réifiés à l'extrême. L'humain a beau prêter son apparence et ses sentiments à la machine, il le fait par l'intermédiaire du jouet: le posthumain se voit de la sorte réduit à l'état d'ersatz au destin régi par des dieux dont il ne peut que supputer l'existence ${ }^{13}$.

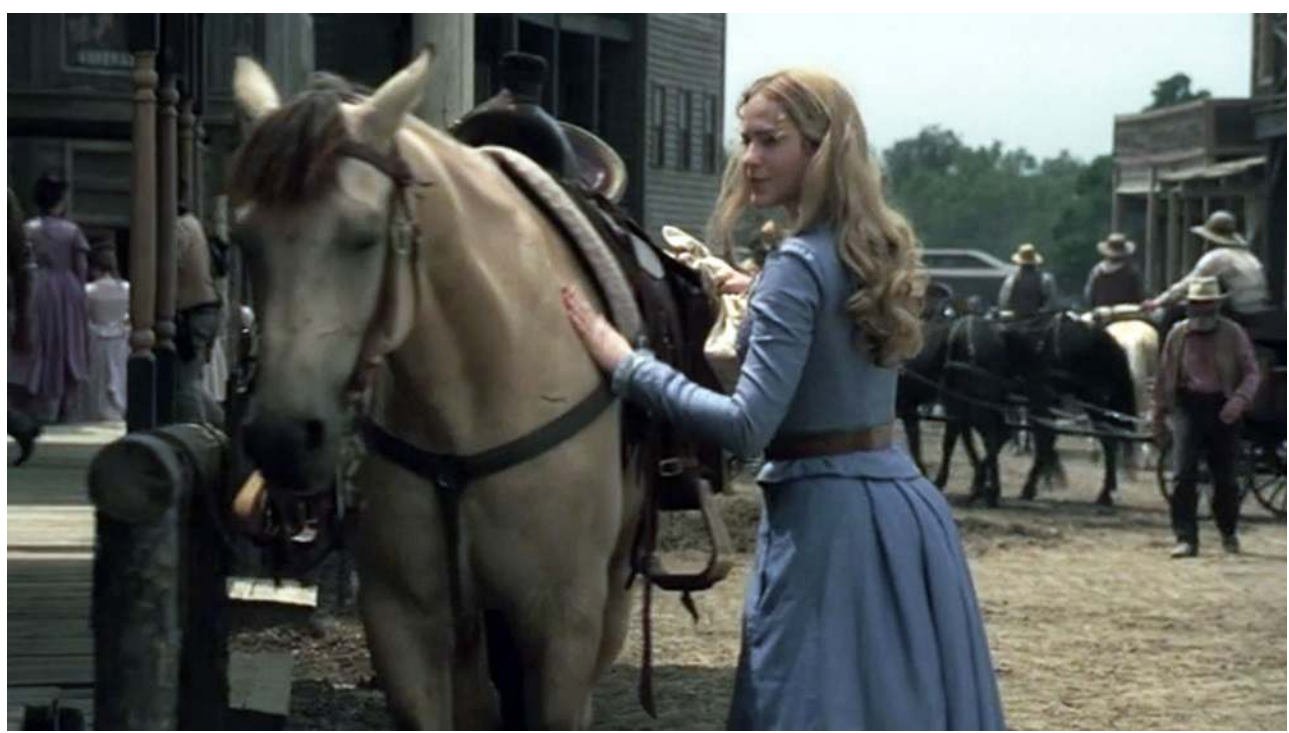

Fig. 4 : Dolores dans sa longue robe bleue (S01E01)

Même quand Dolores est invitée à (enfin) changer de tenue pour participer au détournement d'un chargement de nitroglycérine, c'est en des termes directifs et machistes peu flatteurs : «La blondinette va devoir changer de tenue ${ }^{14}$ » (S01E05). De sa robe bleue au romantisme pour le moins anachronique dans un milieu d'une telle sauvagerie, la jeune femme passe à un pantalon rentré dans de hautes bottes en cuir, maintenu par une épaisse ceinture et accompagné d'un étui à revolver extérieur, d'une chemise rayée aux manches retroussées, d'un foulard prêt à masquer son doux visage et d'un chapeau vert kaki censé la fondre dans le décor (Fig. 5). Un changement de style vestimentaire si brutal que, cette fois-ci, c'est elle qui paraît anachronique dans une tenue hypermasculine en totale inadéquation avec sa caractérisation initiale. Et quand bien 
même on a tendance à considérer, en langage scénique, que «l'habit fait l'acteur » et l'aide à entrer dans la peau de son personnage, Dolores semble pour sa part bien en peine de se fondre dans cette nouvelle carapace et de convertir ce qui s'apparente pour l'heure à un déguisement en tenue de tous les jours.

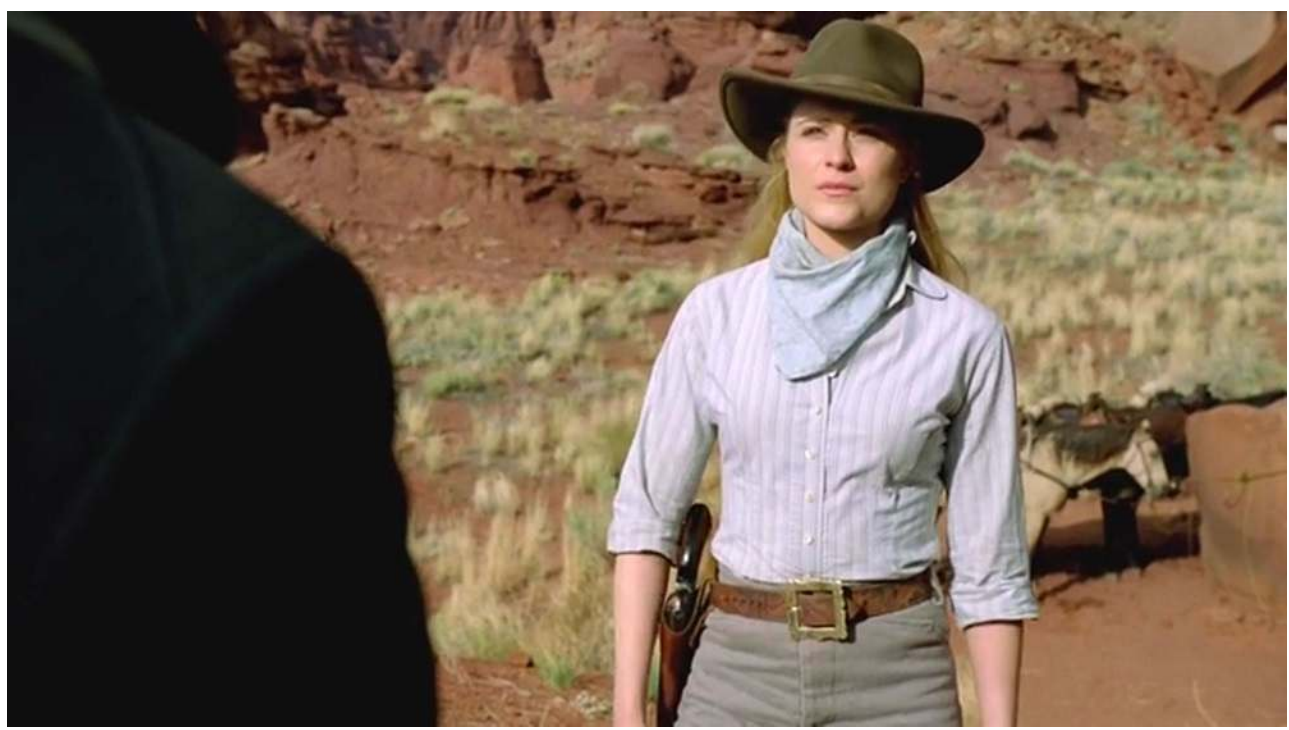

Fig. 5 : Dolores en tenue de bandit de grand chemin (S01E05).

C'est alors qu'entre en jeu la représentation sexuelle : non pas, classiquement, comme un motif d'accomplissement permettant à une jeune fille pure de quitter l'adolescence et de débloquer certains verrous de son existence (les lendemains de sa première fois avec William se révéleront d'ailleurs pour Dolores une bien cruelle désillusion, celui qu'elle prenait pour l'«amour de sa vie » devenant par la suite son persécuteur et même son violeur), mais comme un repoussoir l'incitant à se prendre en main et à retirer l'étiquette de proie sans défense qu'elle porte sur le front. Comme nous allons le constater, Dolores ne prend en effet nullement part à l'orgie de Pariah dans laquelle l'embarque de force le peu recommandable Logan : c'est au contraire en essayant d'y échapper qu'elle se révèle à elle-même et connaît son premier véritable instant érotique. Il serait dès lors tentant de tirer des conclusions hâtives pour conclure que la luxure et la débauche déployées par cette scène à l'esthétique ostentatoire de softporn sont "gratuites", mais ce serait oblitérer la pression environnante que subit Dolores au moment de prendre un chemin de traverse auquel rien ne la prédestinait. Sans être de nature sexuelle, son climax n'en naît en effet pas moins de l'hypersexualisation d'une société sans foi ni loi à laquelle elle refuse de se soumettre plus longtemps ${ }^{15}$.

\section{Une orgie aux (faux ?) airs de clip sexuel}

La scène d'orgie s'ouvre par un lent panoramique vertical découvrant, en une languissante plongée au cadre cerné de bougies rouges parfaitement symétriques, la présence de Logan, William et Dolores au cœur d'un parterre de fornicateurs au corps glabre recouvert d'une dorure luisante évoquant les dieux de l'Égypte antique (Fig. 6). 


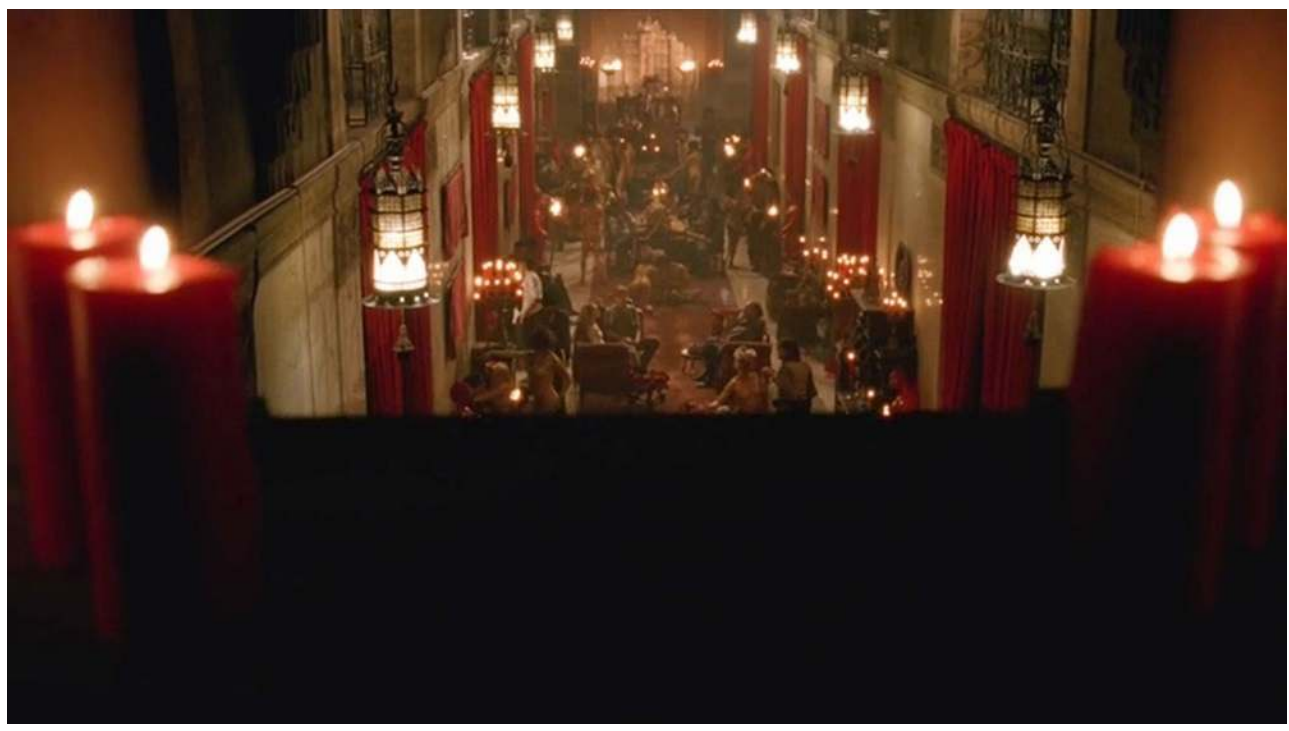

Fig. 6 : Panoramique vertical sur le parterre orgiaque (S01E05)

17 Un plan rapproché, coulissant de gauche à droite, nous signale par le changement de réglage du point et le positionnement de Dolores au centre de sa trajectoire la captivité manifeste de cette dernière, assise de part et d'autre d'inconnus (dont on ne sait plus très bien distinguer les hôtes robotiques des visiteurs humains) qui se caressent et s'accouplent avec une palpable délectation (Fig. 7 à 9).

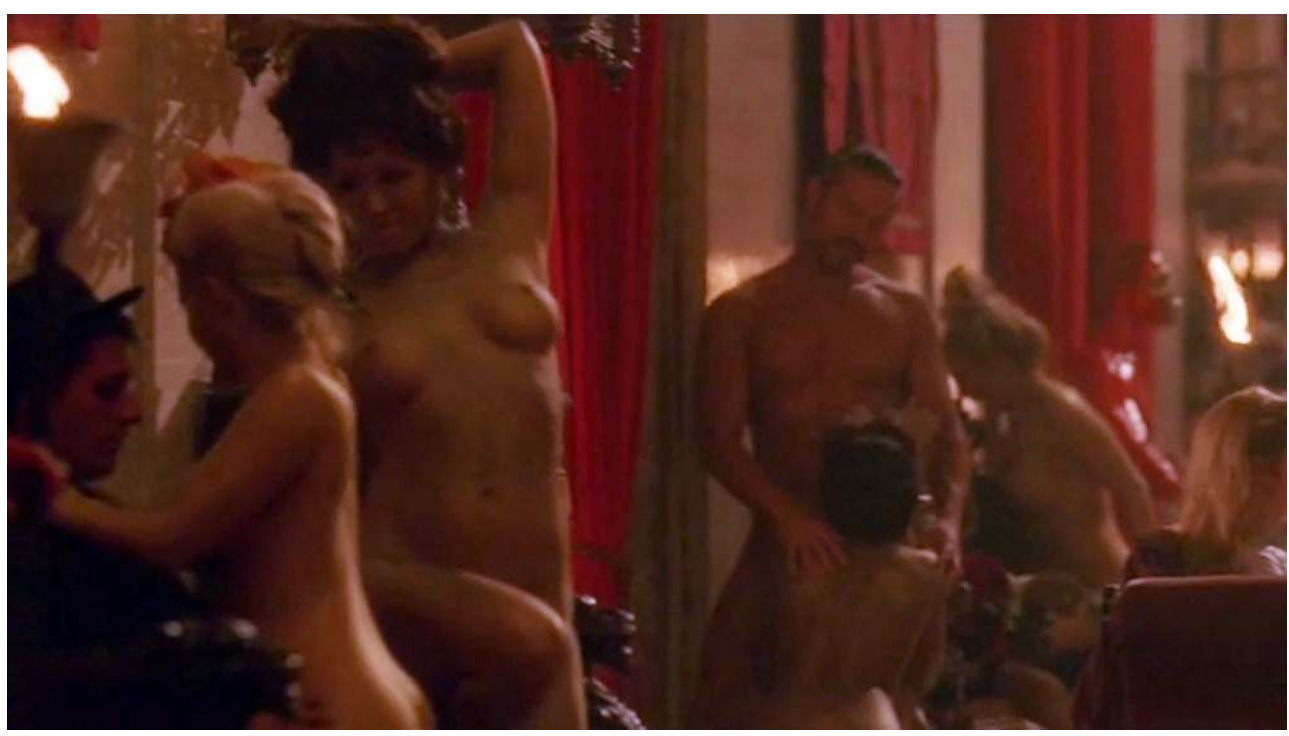



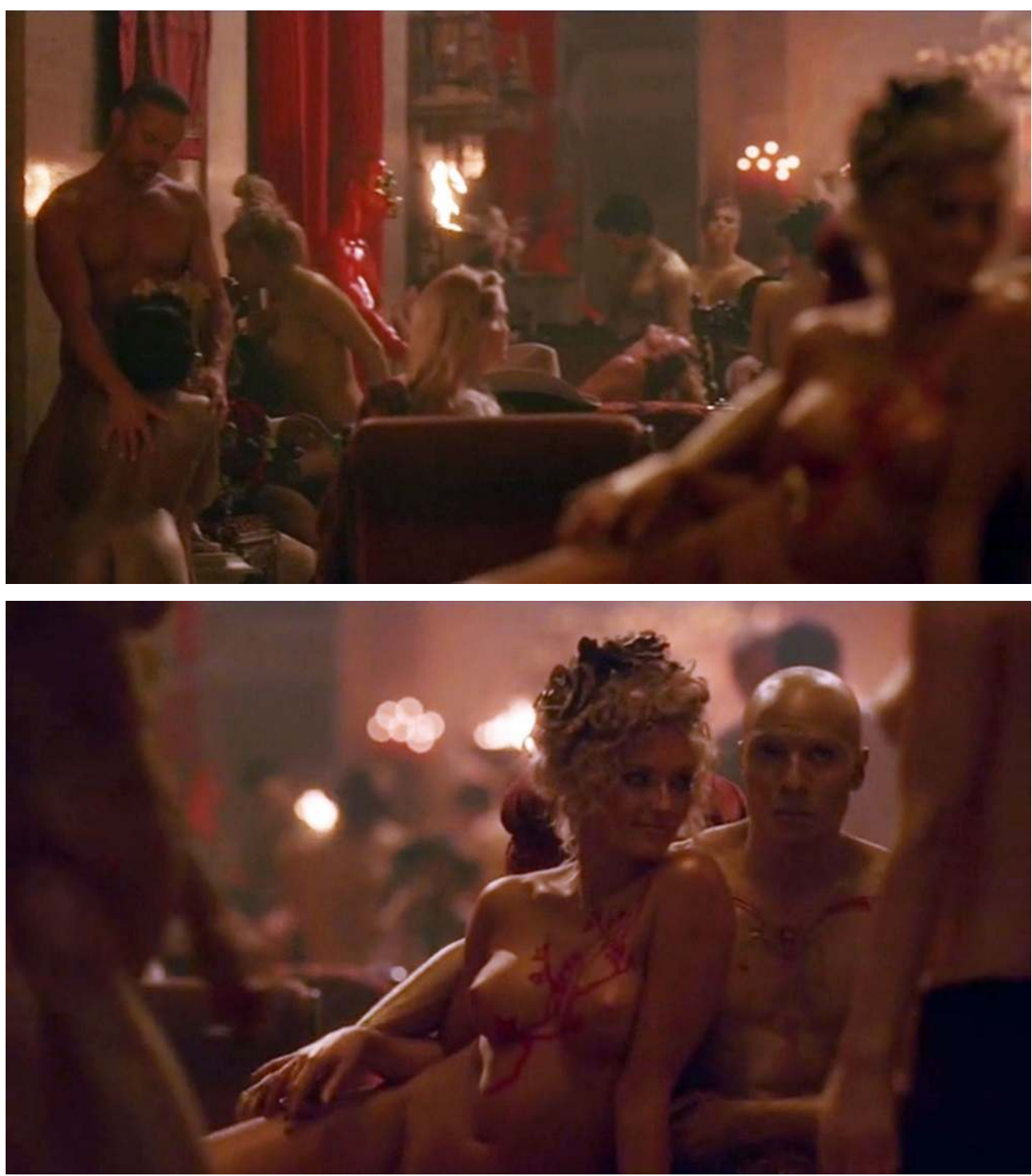

Fig. 7, 8 et 9 : Panoramique horizontal articulé autour de Dolores (S01E05).

18 Éclairées par une profusion de torches, de bougies et de lanternes suspendues, bordées de longues draperies rouges et de corps statufiés recouverts d'une peinture du même ton (comme si la luxuriance de l'étoffe murale avait déteint sur leur chair devenue à son tour décorative), les agapes qui s'ensuivent s'appliquent à remplir le cahier des charges de l'orgie filmée selon les codes traditionnels du softporn: fellations, baisers lesbiens, contorsions goulues, attouchements lubriques, masturbations solitaires ou en groupe, étreintes libertines s'enchaînent sous nos yeux en de brefs mais évocateurs plans fixes occasionnellement mus, tel le plan d'ouverture, par un léger panoramique de bas en haut.

19 La mélopée profonde et entêtante des violons accompagnant la scène rapproche un peu plus celle-ci de ce que Linda Williams nomme des «interludes sexuels en musique» (même si, en l'occurrence, la musique ne recouvre pas entièrement mais se superpose aux gémissements de plaisir des convives). Pour en attester, référons-nous à la définition qu'en donne la chercheuse américaine :

Ces montages lyriques - lorsque la musique prend de l'ampleur à mesure que la narration ralentit - ont permis l'apparition d'une forme acceptable de connaissance charnelle dans le cinéma américain mainstream. L'alliance de la 
musique et du sexe, opposée à la présentation d'actes sexuels peu ou nonaccompagnés de musique, joue un grand rôle dans l'histoire des représentations cinématographiques de la sexualité. Comme les baisers des films muets ou sonores ne se donnaient jamais sans envolée musicale, la connaissance charnelle est quasi toujours accompagnée de musique dans les films de l'après-Code ${ }^{16}$.

Dans le cas qui nous intéresse, cet accompagnement musical ne s'interrompt toutefois pas lorsque, d'un plan fixe sur Logan, suivi d'un travelling avant délaissant les créatures délurées du premier plan pour se concentrer sur les protagonistes réunis dans leur dos, la narration en vient à reprendre son cours (Fig. 10 et 11). Tant musicalement que visuellement, la traditionnelle coupe qui, dans le film érotique ou pornographique, sépare la pseudo-narration préliminaire de la scène de sexe à proprement parler cède ainsi le pas à un glissement intriquant subtilement attraction et narration.
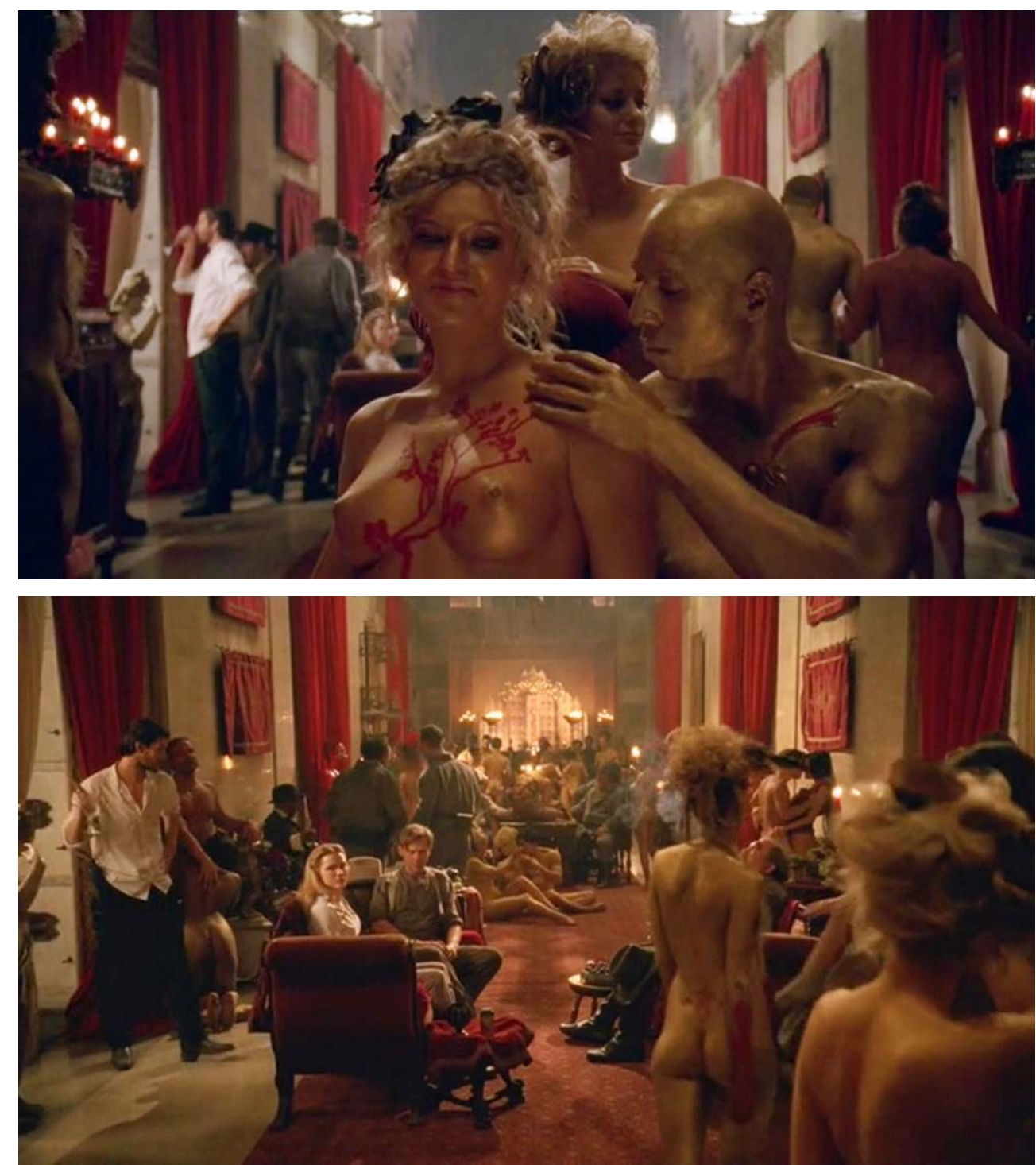

Fig. 10 et 11 : Glissement de l'attraction vers la narration (S01E05).

21 Ce qui m'amène à relever une deuxième entorse à la norme établie par le cinéma $\mathrm{X}$ industrialisé : ici, ce mouvement ne s'effectue pas de la narration vers l'attraction, mais dans le sens inverse. Mécaniquement, l'enjeu qui consiste d'ordinaire (pour les 
personnages comme pour les spectateurs) à atteindre la jouissance opératoire du dispositif pornographique se voit dès lors supplanté par la préfiguration d'une autre forme d'épanouissement, plus inattendue donc plus signifiante. Pour Dolores, qui se croyait définitivement contrainte d'accepter son triste sort de victime expiatoire incapable de se défendre par elle-même, il est temps de redistribuer les cartes et de ne plus accepter de tendre docilement l'autre joue.

\section{Fuir le sexe pour mieux se l'approprier}

Tentant d'échapper à une atmosphère de débauche devenue irrespirable, la jeune femme profite d'un règlement de comptes entre William et Logan pour s'éclipser. Elle parcourt alors un long couloir aux renfoncements libidineux, puis trouve refuge dans une alcôve secrète où se tapit une mystérieuse diseuse de bonne aventure. Celle-ci l'invite à tirer une carte (qui porte, comme de bien entendu, le symbole du labyrinthe), avant de se métamorphoser hors-champ en double de Dolores, vêtue de sa longue robe bleue originelle. Dans le plan en miroir qui suit apparaissent, assises l'une en face de l'autre dans une posture quasiment identique, Dolores la «fille d'éleveur au cœur pur » et Dolores la "justicière (pas encore tout à fait) intrépide » (Fig. 12). La mutation est en marche, mais il reste une étape décisive à franchir : celle qui consistera pour l'hôtesse à s'approprier son nouveau rôle ou, s'agissant d'une androïde réduite à n'interpréter qu'un seul personnage jusqu'à nouvel ordre, à faire de son nouveau costume une véritable seconde peau.

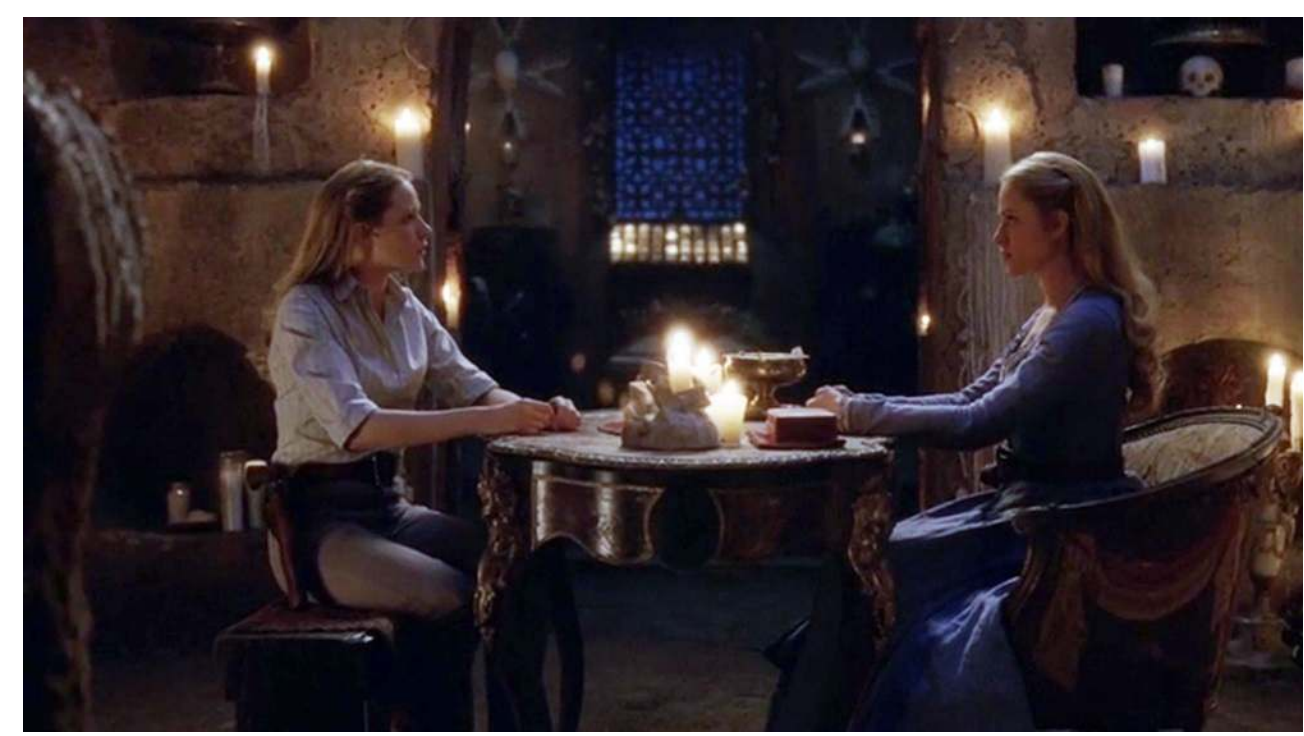

Fig. 12 : Plan en miroir des « deux » Dolores (S01E05).

Sur la voie de cet accomplissement personnel, Dolores se voit doublement confrontée à une expérience traumatique de manipulation du corps. Le sien d'abord, qu'elle « détricote » en tirant sur un fil qui dépasse étrangement de son avant-bras droit, avant de s'apercevoir qu'il s'agissait d'une simple (mais terrifiante) hallucination; celui des siens ensuite (même si elle ne fait guère de différence entre hôtes et humains), victimes de la vilenie de bandits de grand chemin qui les vident de leur sang pour remplacer celuici par de la nitroglycérine macabrement clandestine. Dans les deux cas, l'infiltration de la chair robotique - sans commune mesure avec l' «hygiénique combinaison de plongée ${ }^{17}$ » que déplore Jean-Sébastien Chauvin au sujet de la (non-)corporéité des protagonistes du 
remake live de Ghost in the Shell (2017) - renvoie les spectateurs que nous sommes à l'exploitation sans vergogne de la machine par l'homme, dont Westworld (le parc comme la série du même nom) a fait un loisir de luxe réservé à une élite embourgeoisée en manque de sensations fortes. Mais pour Dolores, il s'agit plus innocemment d'interroger la nature profonde du corps au sens générique du terme, surexposé au-dehors - car pendant ce temps-là, l'orgie continue de battre son plein -, mais toujours nimbé de mystère au-dedans.

La révélation finale intervient lorsque, cernés par des confederados s'estimant (à juste titre) floués, William et Dolores se retrouvent sur le point de payer de leur vie la mystérieuse disparition du chargement de nitroglycérine. Poussée par son compagnon chevaleresque à fuir pendant qu'il livre son dernier combat, la « demoiselle en détresse » désobéit alors pour la première fois de son existence à un ordre et abat la bande de malfrats d'une salve de tirs dont elle ne se serait jamais crue capable. Filmé en gros plan après avoir fait retentir quatre coups aussi secs qu'imprévisibles, son revolver nous apparaît dans la foulée comme un prolongement naturel de sa main - alors que, de son propre aveu, elle osait à peine le sortir de son étui quelques heures plus tôt (Fig. 13).

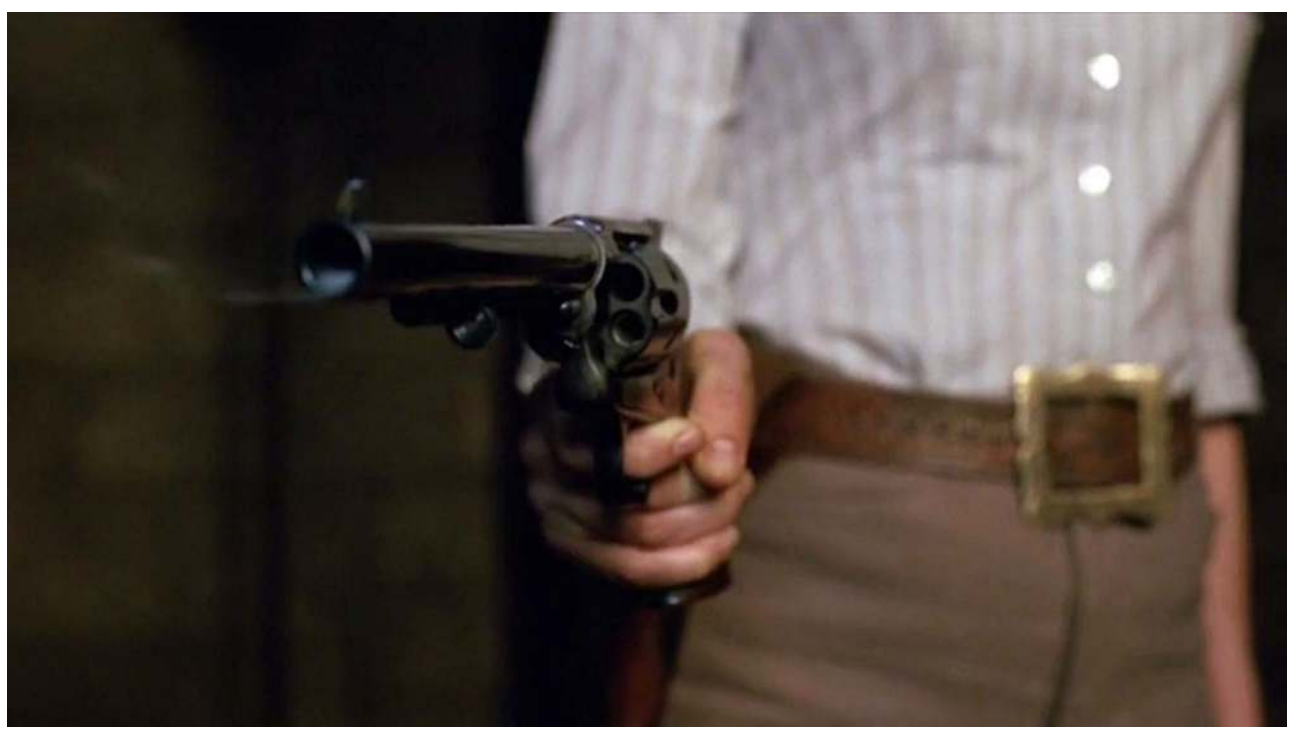

Fig. 13 : Gros plan du revolver de Dolores (S01E05)

Ses bottes de cuir montantes, son pantalon d'exploratrice, sa chemise aux manches retroussées, n'ont dès lors plus rien d'anachronique : ils habillent au contraire le corps idoine, comme on dit d'un rôle qu'il est «fait pour cet acteur ». Dolores finit ainsi par prendre possession de son propre corps (tenue vestimentaire incluse), non en le mettant à nu pour ressembler aux créatures délurées qui forniquent autour d'elle tout au long de l'orgie dont elle finit par s'évader, mais en le fondant dans la peau d'un personnage hors script dont elle ne parvient à prendre la pleine mesure que rétroactivement. Elle n'atteint donc pas l'orgasme par le biais classique d'une première expérience sexuelle; elle expérimente sexuellement la prise en main de son destin et de son nouveau statut d'hérö̈ne capable de se défendre par elle-même.

C'est ce qu'illustre la mise en scène en faisant se succéder - avec, dans l'interstice, un contrechamp annonciateur sur William apparaissant dans le viseur de sa future adversaire (Fig. 14) - un lent panoramique de bas en haut et une plongée à hauteur de plafond sur Dolores (Fig. 15 et 16). Soit une grammaire visuelle reproduisant précisément 
les choix esthétiques qui avaient servi à capter la langueur des corps fiévreux s'ébattant en ouverture de scène.

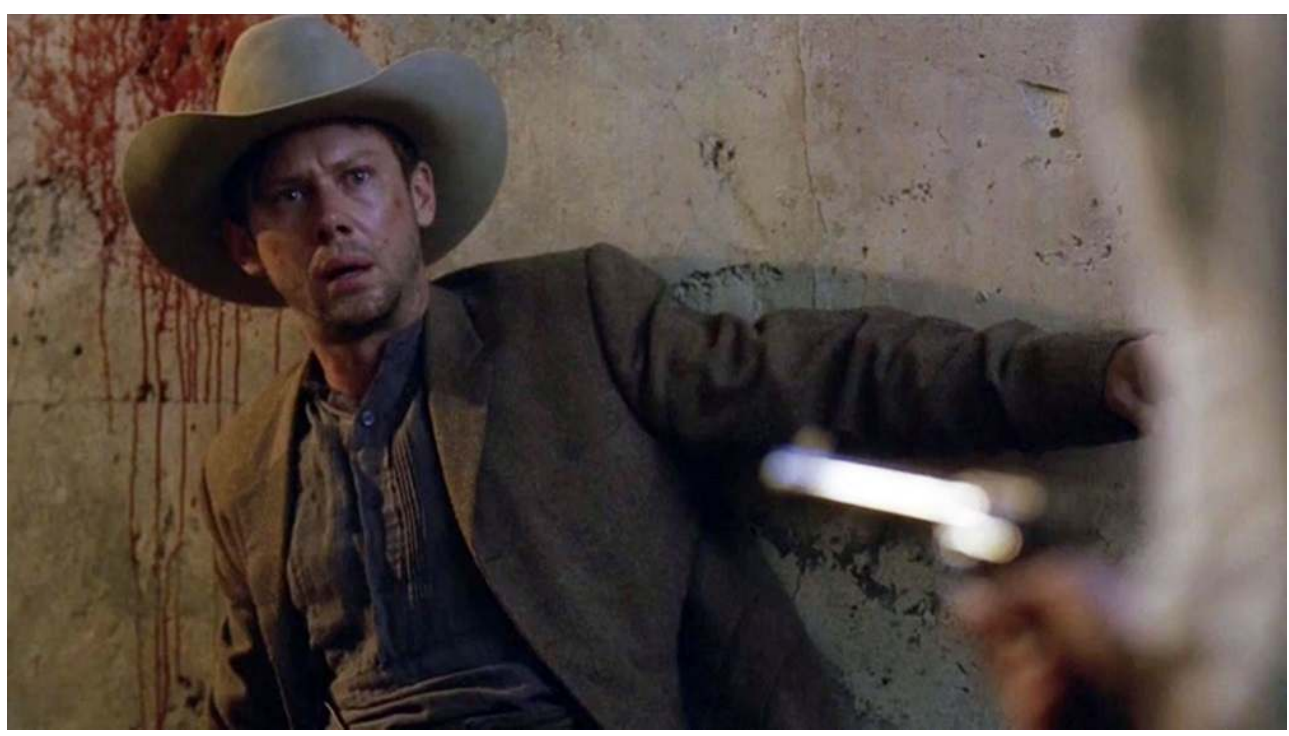

Fig. 14: William dans le viseur de Dolores (S01E05)

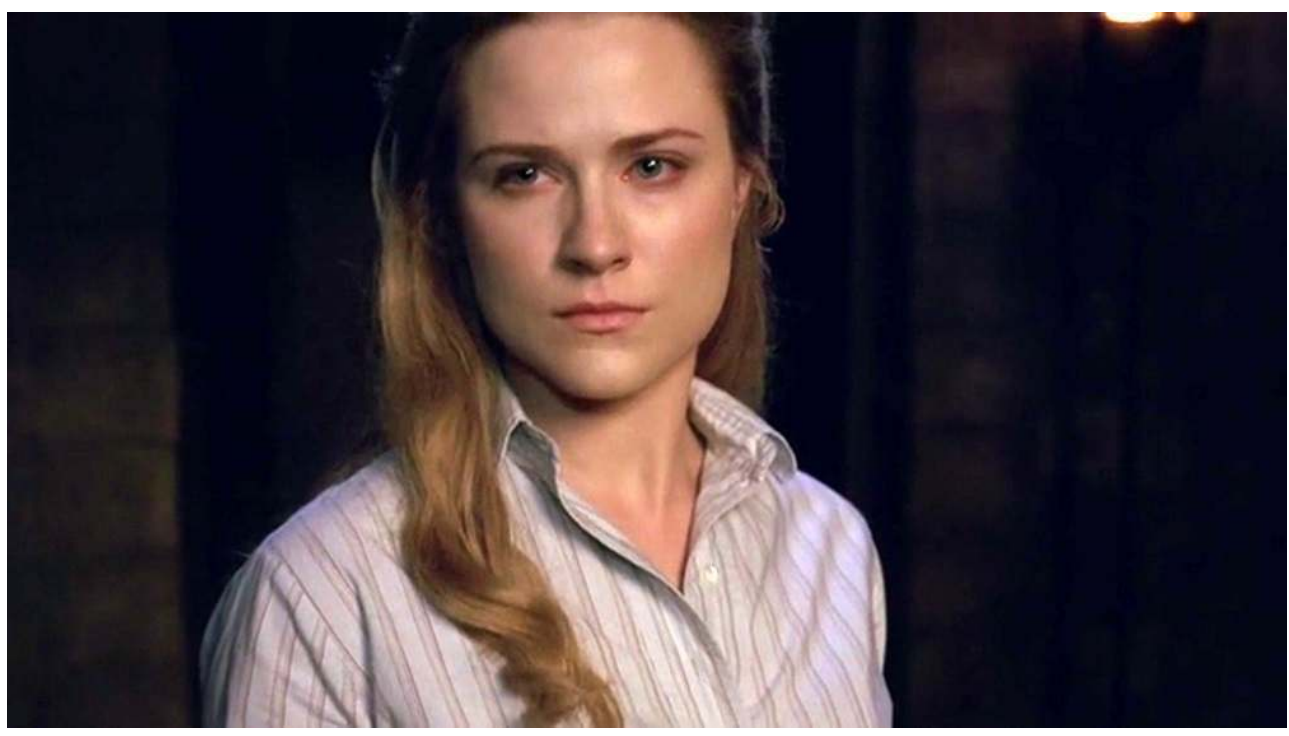

Fig. 15 : Panoramique de bas en haut sur Dolores (S01E05). 


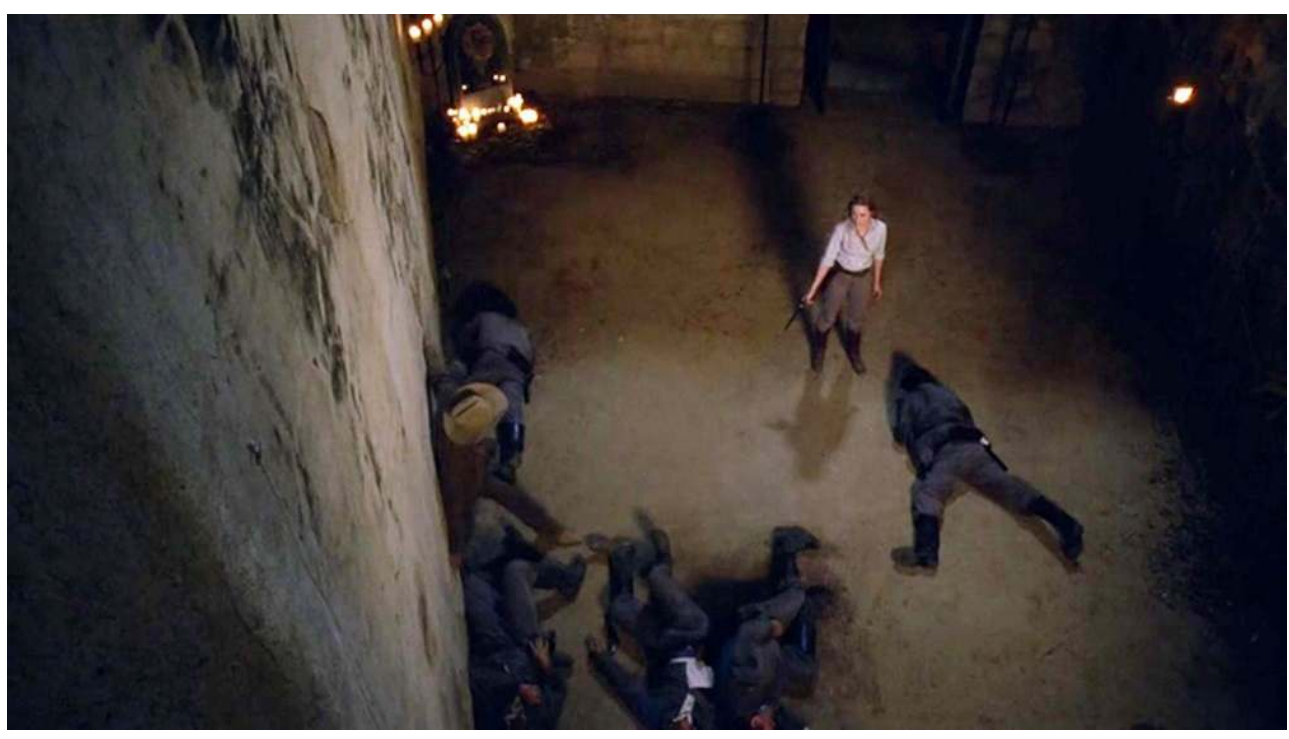

Fig. 16 : Plongée à hauteur de plafond sur Dolores (S01E05).

Au diapason de cette métonymie, l'accompagnement musical procède par effet de rupture comme s'il tenait à nous signaler le changement qui s'opère au même moment en Dolores. La musique s'interrompt juste avant que n'éclatent les quatre coups de feu tirés par cette dernière; puis elle reprend son cours, précisément au moment où survient le panoramique vertical synonyme de "petite mort» pour l'ancienne ingénue devenue pistolero. Cette fois-ci, la coupe entre narration et attraction caractéristique du film pornographique se signale donc distinctement, tant par la monstration explicite d'un gros plan de revolver - dont la symbolique phallique semble décidément inusable - que par la diffusion d'un thème musical opératique insistant sur la notion de " performance » de l'hôtesse libérée de ses chaînes.

Tout est par conséquent mis en œuvre pour nous convier à une scène de sexe sans le sexe (au sens traditionnel du terme) - l'ultime ironie de cette translation autoréflexive étant que le sexe est par ailleurs omniprésent, que ce soit dans la scène que je viens d'analyser ou, plus généralement, sur HBO, dont la réputation (quelque peu surfaite quand on l'étudie dans le détail) de chaîne particulièrement portée sur la « chose " n'est plus à faire ${ }^{18}$. Mais Westworld ne se contente pas de placer une oie blanche au cœur de la débauche (post)humaine pour mieux déjouer nos craintes de spectateur habitué à redouter le pire ${ }^{19}$. La série va plus loin en renouvelant et en reconfigurant l'expérience orgasmique de son autre protagoniste principale : Maeve, mère maquerelle à la fois hyper- et désexualisée, dont nous allons voir dans la seconde partie de cet article qu'elle incarne une nouvelle forme d'empowerment féminin. Non pas de celles qui laissent le sexe aux autres pour se concentrer sur les "affaires sérieuses" (comme si le sexe n'était pas en soi une affaire sérieuse), mais de celles qui observent le sexe « de l'intérieur » pour mieux en détourner les conventions établies et les tics de représentation. Si chaque scène de sexe raconte une histoire, il nous est ici rappelé fort à propos que l'histoire en question n'est pas forcément à caractère sexuel. Même si l'effet diégétique produit est le même. 


\section{Un « fix » aperceptif pour atteindre le septième ciel : Maeve et l'expérimentation d'une nouvelle forme de jouissance}

D'un côté, nous avons Dolores: une jeune femme blanche, pure, innocente, inexpérimentée, idéaliste, qui s'évertue à voir la "beauté du monde " plutôt que «sa laideur et son chaos ${ }^{20} »($ S01E01). De l'autre, Maeve : une tenancière de maison close plus mûre, métisse, réifiée, usée par la vie et les passes qui s'y sont enchainées, revenue du sexe et totalement désabusée sur l'avenir de l'humanité dont elle croit faire partie. En termes scénaristiques, nous pourrions postuler de prime abord que Maeve incarne une potentielle antagoniste de Dolores - le second plan dans lequel elle apparaît, devisant sur le rejet des faux-semblants du commerce sexuel auquel se livrent les prostituées du Mariposa, marque d'ailleurs visuellement le contraste lorsque, par le biais d'un léger panoramique de droite à gauche conjugué à une rapide mise au point de la caméra, s'incarne à travers une fenêtre donnant sur l'extérieur la présence angélique de Dolores sortant de l'épicerie (commerce n'ayant a contrario rien de sexuel), comme une manière d'attester formellement la distance morale qui la sépare de Maeve (S01E01, Fig. 17 et 18).

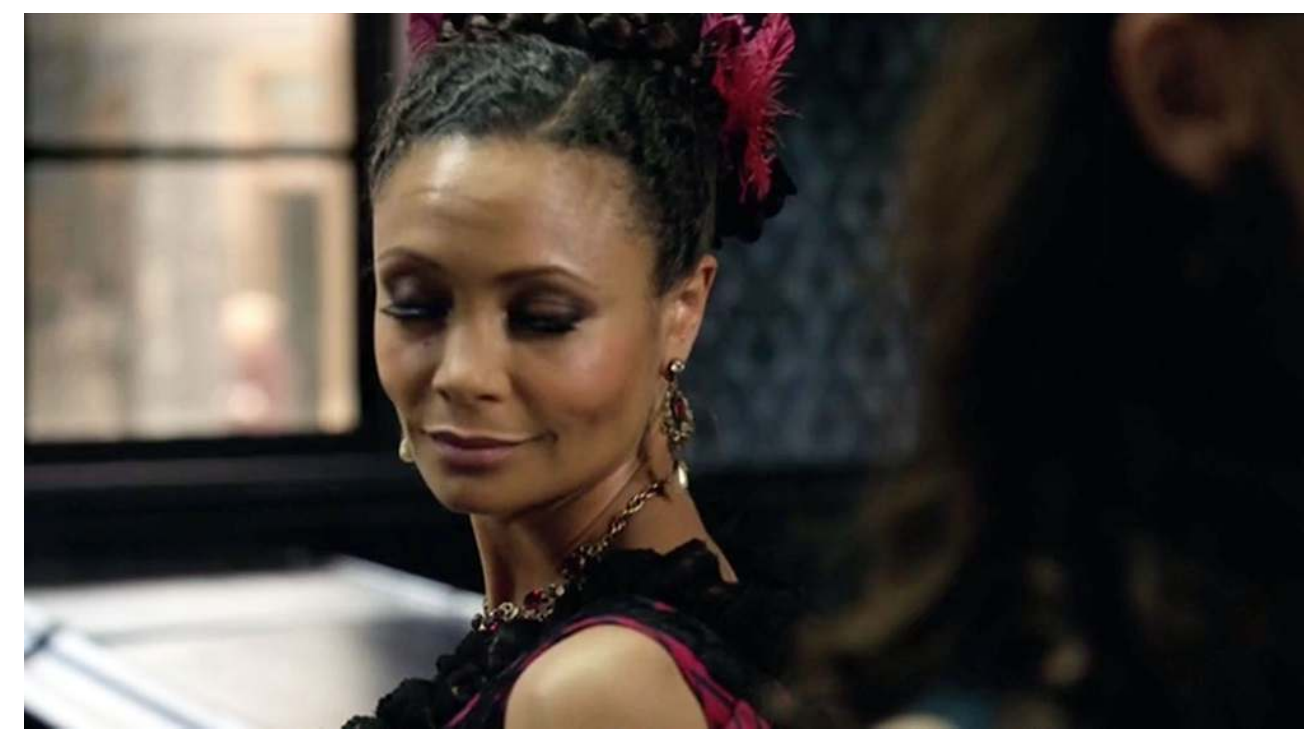

Fig. 17 : Maeve au premier plan dans le Mariposa (S01E01). 


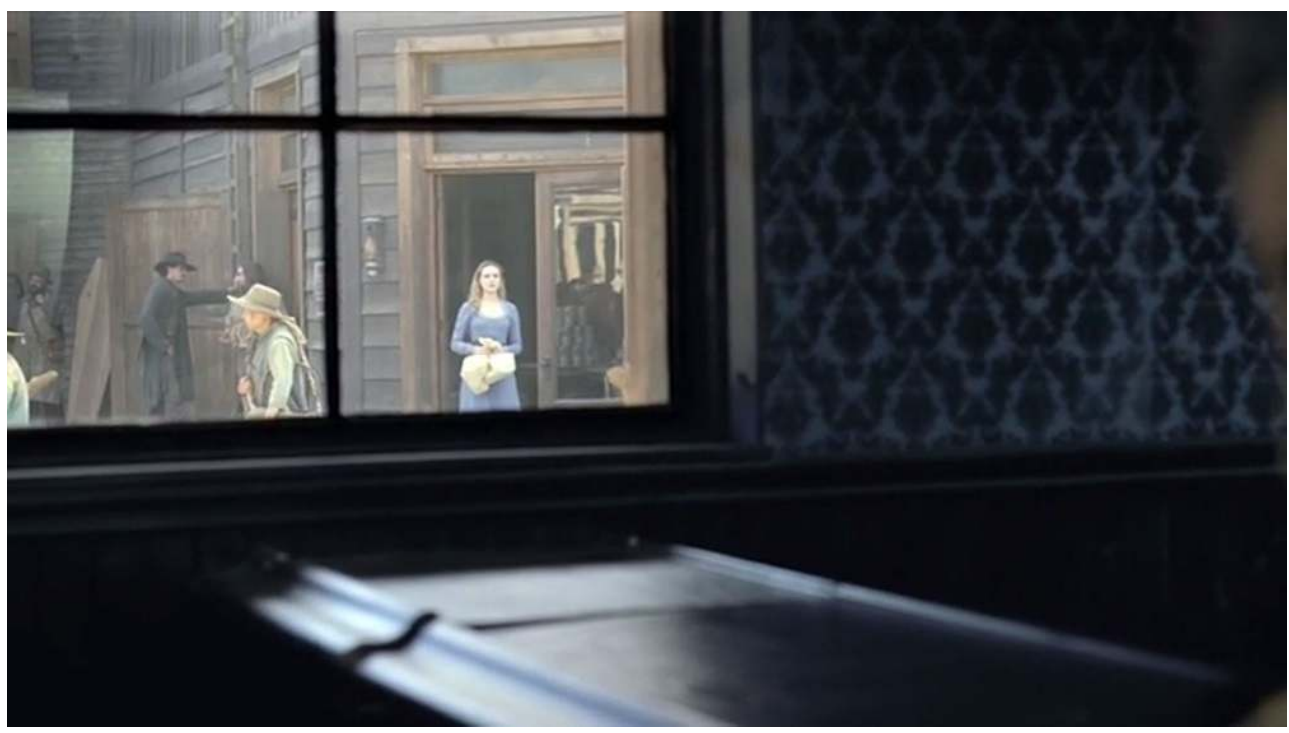

Fig. 18 : Dolores sortant de l'épicerie au second plan (S01E01).

Mais de la même manière que l'homme en noir se révélera plus lié que nous ne le présagions à sa Némésis en puissance (à savoir William), de nombreuses rimes visuelles s'appliquent à nous présenter Maeve comme l'autre face du Janus posthumain de Westworld : une face tournée vers l'avenir (malgré un passé cruel qui ressurgit de manière chronique par de violents accès de "rêverie" traumatique), alors que son entité originelle reste pour sa part inexorablement coincée dans le passé. Après l'éveil - somme toute illusoire, mais nous ne le comprendrons que par la suite - de Dolores dans Contrapasso (S01E05), c'est en effet au tour de Maeve de sortir de sa propre torpeur dans l'autre épisode charnière de la saison, ironiquement intitulé The Adversary (S01E06). À l'instar de Dolores, l'androïde apparaît en début d'épisode face caméra, cadrée en plan cravate, allongée sur son lit au petit matin, prête à débuter une nouvelle journée en majeure partie identique à toutes celles qui ont précédé depuis son entrée dans l'âge adulte (Fig. 19 et 20). Car sa routine n'est pas seulement celle d'un parc rejouant sempiternellement (à son insu) les mêmes attractions : c'est aussi celle d'un personnage dont l'existence a fini, avant même d'avoir commencé, par tourner en boucle.

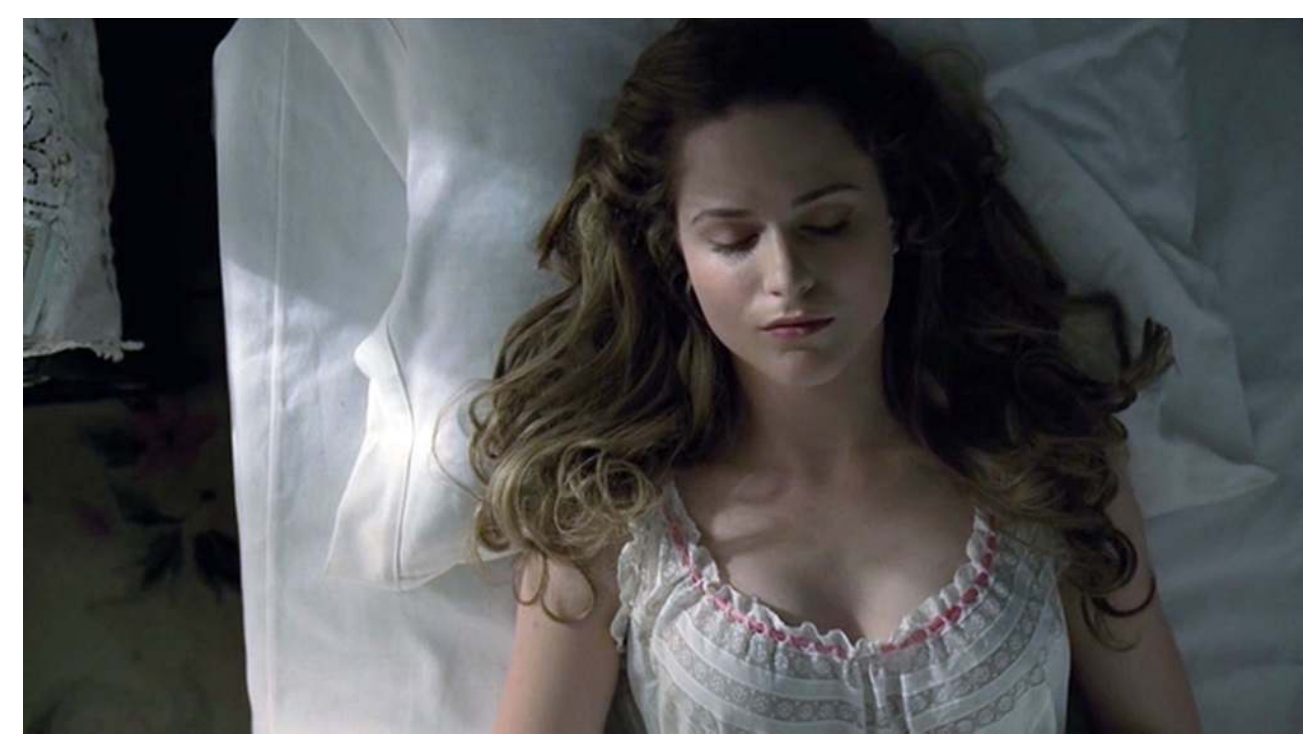




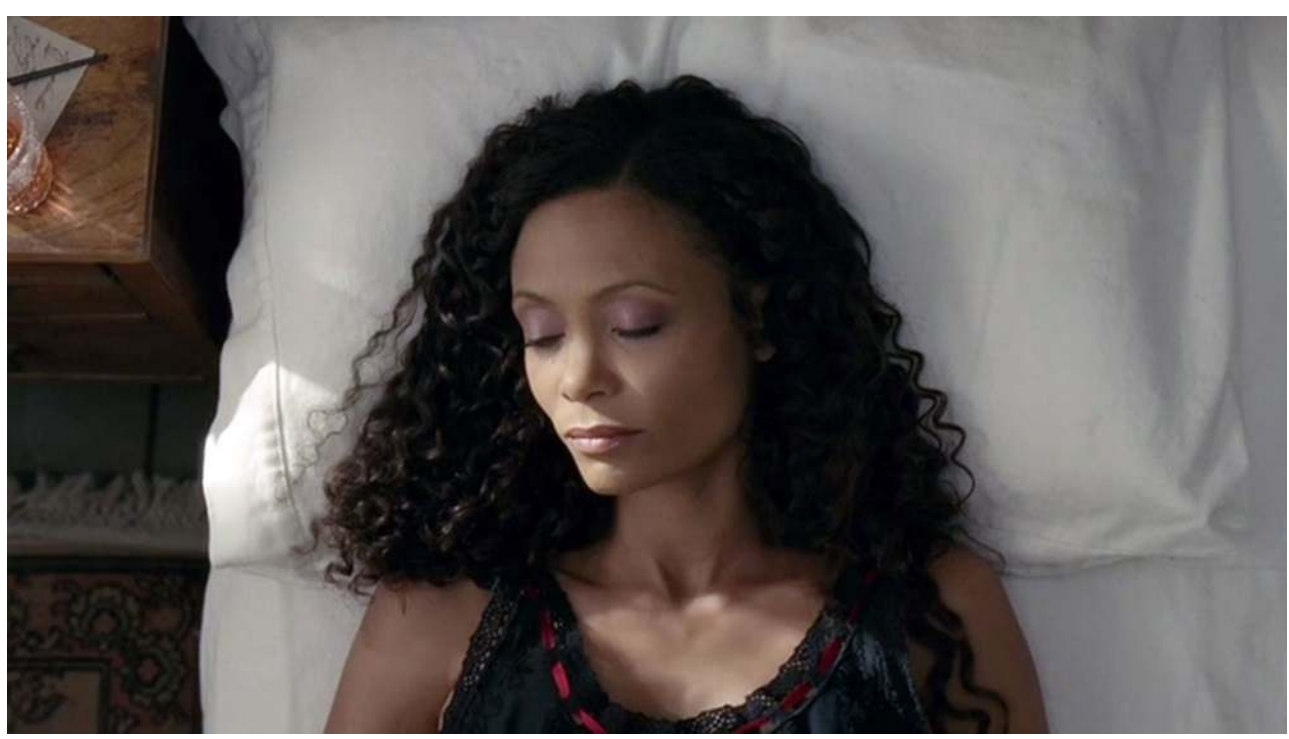

Fig. 19 et 20 : Dolores et Maeve au réveil, face caméra (S01E01 et S01E06).

31 Pour sortir de sa cage, ce moineau-là (prolongement évident de celui auquel Felix parvient à redonner vie dans les derniers instants de l'épisode précédent) aurait tout intérêt à jouer de ses charmes et à mener par le bout du nez les visiteurs rustres et crasseux qui se succèdent au Mariposa. Autre option scénaristique: elle pourrait s'émanciper par un retour sur le « droit chemin » de la vertu et du sexe libre, en faisant fi de l'univers ultra-codifié et ultra-genré du western. La série lui offre cependant l'occasion d'opter pour une voie médiane en remodelant son (accablante) expérience sexuelle, plutôt que de la repousser vers sa ligne d'horizon à l'instar du poor lonesome cowboy acheminant sa monture vers de nouvelles aventures. C'est ce qui fait de la création de Jonathan Nolan et Lisa Joy, en dépit de difficultés de production ayant mis en doute la tangibilité de son arrivée à terme, une "série $\mathrm{HBO}$ » à part entière : une série adulte, consciente du caractère fondamental de la question sexuelle aussi bien en termes de devenir que d'agentivité.

\section{L'envers du décor, ou la fin de l'illusion}

Tout comme Dolores, c'est en rencontrant un humain au cœur (apparemment) pur que Maeve entrevoit la possibilité de son émancipation. En témoigne ce gros plan de sa main qui saisit tendrement celle de son « dieu protecteur »- Felix, spécimen atypique parmi les techniciens confinés à la solitude et à la misère sexuelle du laboratoire de Westworld -, en écho féminisé au gros plan de la main de William saisissant celle de Dolores dans l'épisode précédent (Fig. 21 et 22). Nous allons cependant constater que la fiche personnage de Maeve lui impose de nouer avec ce nouveau partenaire sexuel (par procuration) une relation plus intime que celle liant Dolores à William, quoique s'affranchissant à son tour de l'impératif d'accouplement elliptique ponctuant la traditionnelle romance hollywoodienne du type « Boy meets girl ». 


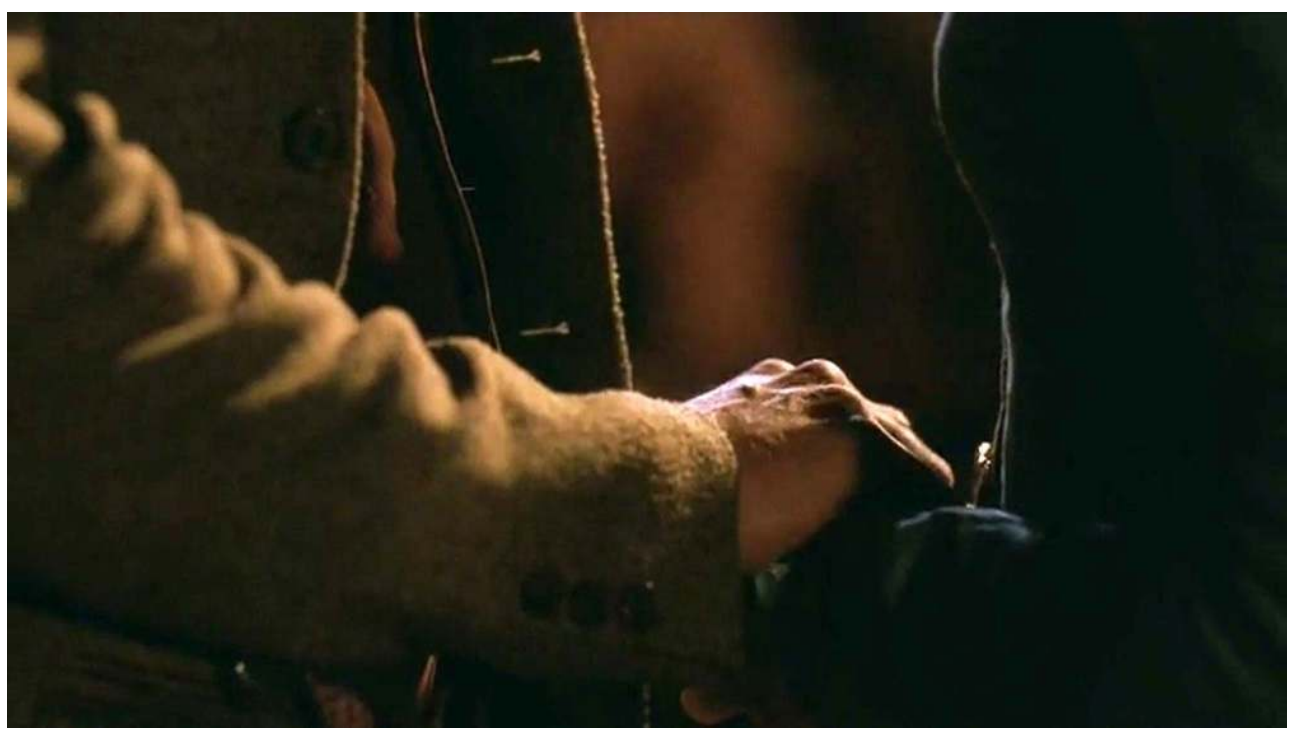

Fig. 21 : Gros plan de la main de William saisissant celle de Dolores (S01E05).

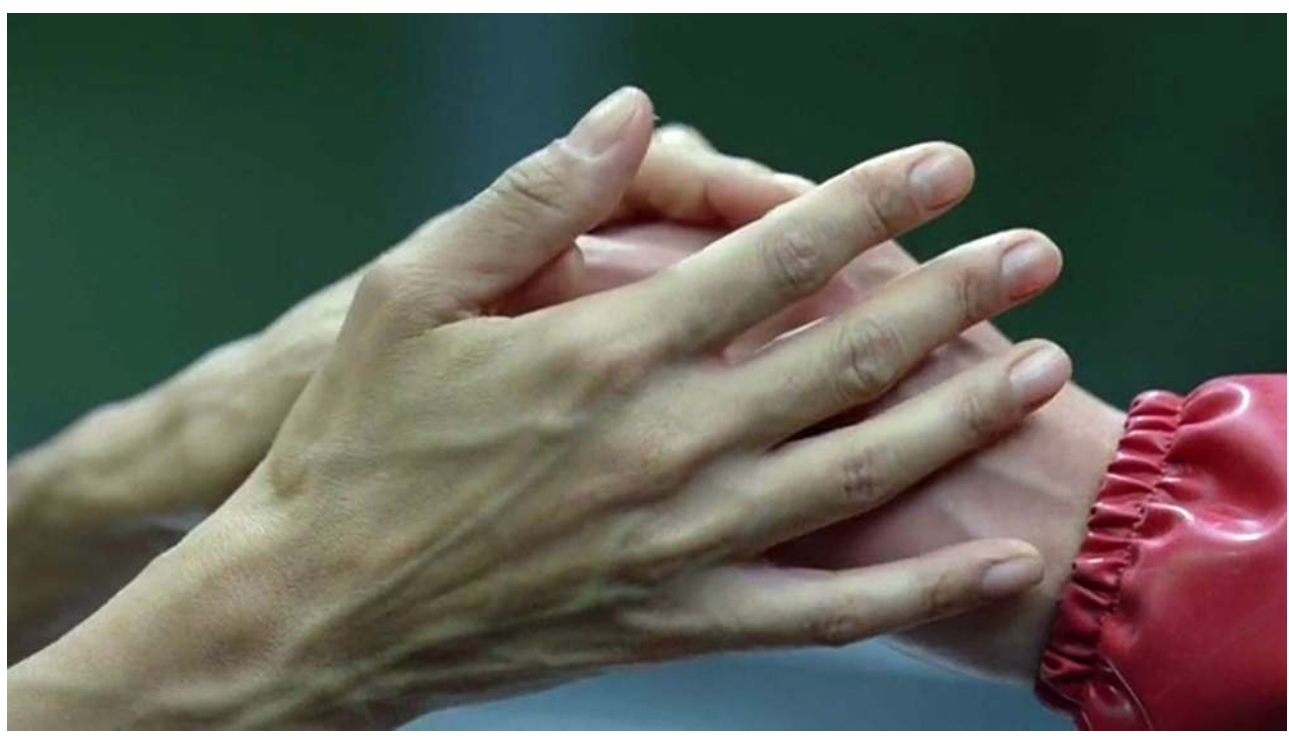

Fig. 22 : Gros plan de la main de Maeve saisissant celle de Felix (S01E06).

Désormais au fait de la vie après la mort qui s'ouvre aux individus de son espèce (aussi indéfinissable soit cette dernière), Maeve pousse un client particulièrement crapuleux à l'étrangler à mort pendant l'acte sexuel afin de renouer le dialogue avec Felix, tout en retournant les agressions dont elle est couramment victime contre les scénaristes de son funeste sort. Encore lui reste-t-il à comprendre précisément les règles du jeu, une fois ce premier seuil franchi. C'est Felix qui lui en livre les grandes lignes, racontant par le menu :

Tout ce que tu fais, tu le fais parce que des ingénieurs t'ont programmée dans ce sens. Tu n'as pas le choix. [...] C'est ton personnage. Tu ne te laisses pas faire. Même quand tu dis non aux visiteurs, c'est préétabli. [...] Tu es sous notre contrôle. Enfin, sous leur contrôle. Ils peuvent te changer à volonté, te faire oublier le passé. [...] Tu peux certes improviser un peu, mais l'essentiel de ce que tu dis a été décidé à l'avance là-haut, comme l'ensemble de ton personnage ${ }^{21}$. qu'il lui fasse visiter l'envers du décor. Telle Dolores dans l'épisode précédent, l'hôtesse 
sous le choc prend, à cet instant, conscience de l'extrême vulnérabilité de ces corps (d'apparence humaine ou animale) que l'on entrepose, démembre et manipule sans soulever la moindre protestation chez leurs « occupants ». Déambulant dans les couloirs de la pseudo-usine à rêves, gravissant ses étages comme autant d'échelons artistiques d'un studio de développement (maintenance, répétitions, conception, promotion), Maeve réalise non seulement que son quotidien n'est qu'une funèbre mise en scène industrialisée, mais en outre que l'outil de travail que constitue son corps ne lui appartient en réalité pas le moins du monde. Quand un torse plâtreux dans lequel on injecte du sang synthétique se met à prendre une teinte saumon et à produire des battements de cœur devant elle, elle se voit confrontée sans le savoir à la formule picturale du Week-end de Jean-Luc Godard (1967) : «C'est pas du sang, c'est du rouge » (Fig. 23 et 24). À la différence près qu'ici, il ne s'agit pas de contrer la violence d'une fiction dérangeante par la force d'une pirouette métonymique, mais au contraire d'endurer la violence de cette fiction par l'excavation de la pirouette métonymique ayant justement servi à l'engendrer. En d'autres termes, c'est parce qu'elle réalise que son sang est du rouge que Maeve semble sur le point de s'évanouir.
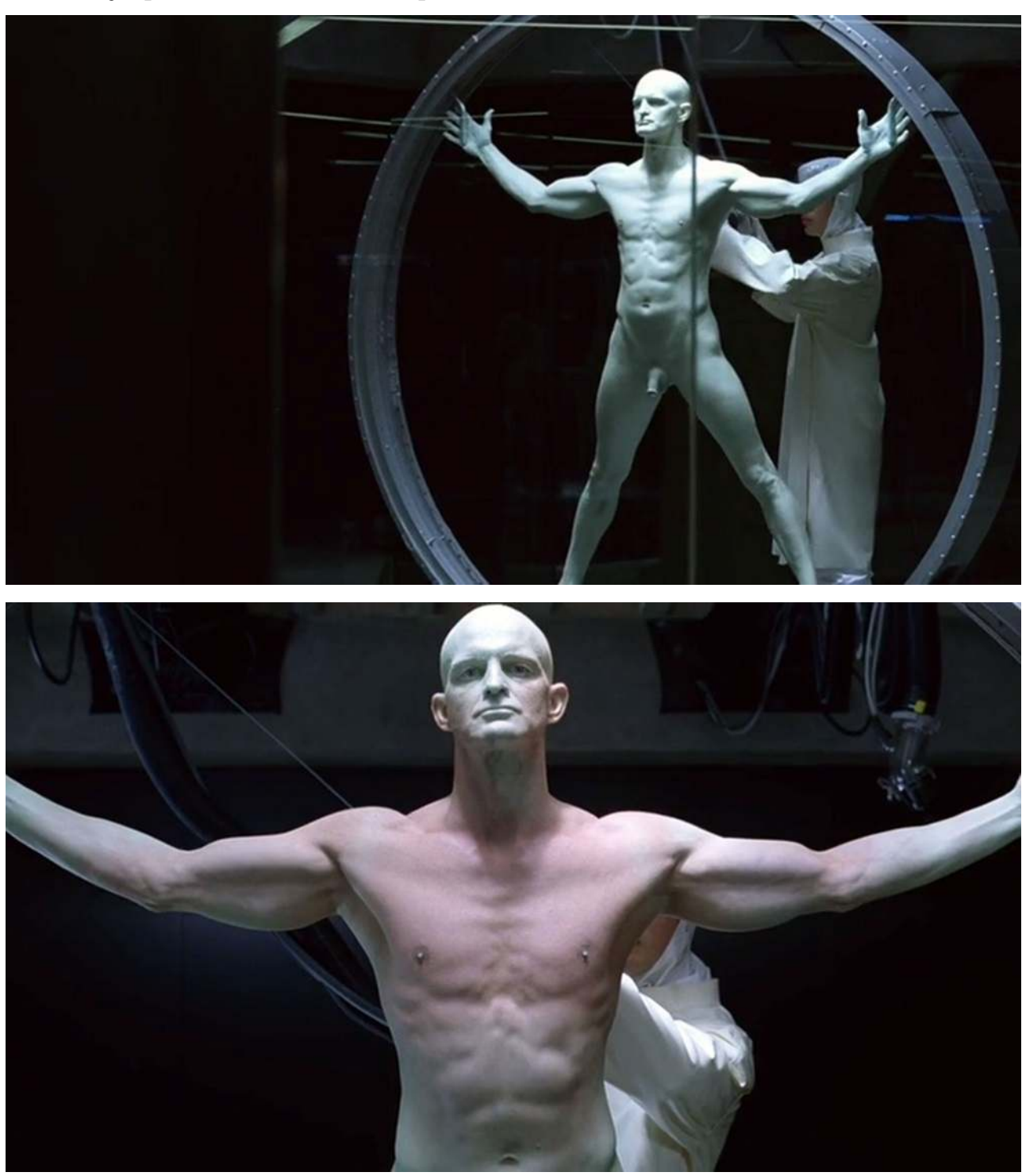

Fig. 23 et 24 : Torse plâtreux prenant vie par la couleur (S01E06). 
Érotisante par la précision de ses cadrages, l'onctuosité de ses travellings et la majesté de ses violons langoureux, cette exploration des coulisses de sa propre vie constitue paradoxalement pour Maeve une épopée transgressive déchirante, en même temps qu'elle interroge notre propre curiosité de spectateur « 2.0 » toujours à l'affût de ce qui se trame de l'autre côté de la caméra. La révélation s'avère aussi fascinante que vertigineuse pour l'hôtesse (qui se sait à présent hôtesse), incapable de détacher le regard du lugubre spectacle de la mort, à la manière de ces automobilistes roulant au pas pour ne rien rater d'une scène d'accident au magnétisme irrésistible. Le paroxysme orgasmique de Maeve ne survient toutefois qu'au moment précis où celle-ci parvient à modifier les règles du jeu et à dicter le changement de sa propre matrice d'attributs ontologiques.

\section{Du plaisir d'obtenir de bonnes notes}

Sylvester, le collègue volubile mais au fond terriblement craintif de Felix, commence par moquer la décision de ce dernier de vêtir Maeve d'une élégante robe en soie noire : «Ça vire au délire hentai ${ }^{22}$ ! s'insurge-t-il en référence aux mangas et aux anime affichant ouvertement leur caractère pornographique (Fig. 25). Se réaffirme ici la perversion qui consiste à jouer à l'hôtesse comme on joue à la poupée, à la nuance près que la déviance ne consiste pas (comme dans le cas de Dolores) à l'habiller de telle ou telle tenue incluse dans la «boite", mais au contraire à la priver de sa nudité synonyme de passage en maintenance. Le dévoilement de ses charmes reste pour l'heure encore inscrit dans le code génétique - ou plutôt, informatique - de Maeve, vouée à satisfaire inlassablement l'appétit sexuel de ces messieurs, qu'elle se trouve dans le parc ou dans son centre de contrôle.

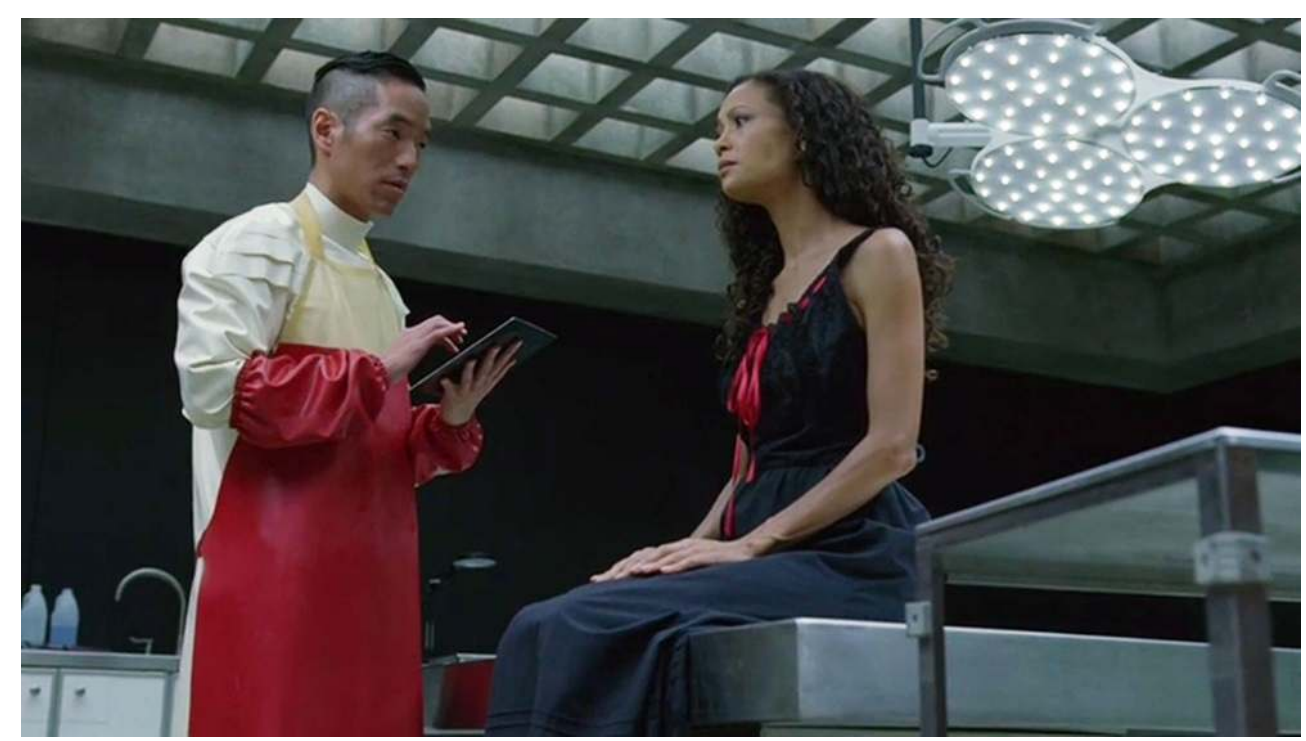

Fig. 25 : Maeve vêtue d'une robe de soie noire (S01E06).

Ce que semble toutefois ignorer Sylvester, c'est que le mot hentai désigne également la «transformation» ou la "métamorphose» dans la langue japonaise. Une méconnaissance qu'il paye au prix fort lorsque Maeve l'attrape par les épaules et menace de l'égorger d'un bistouri si, par le plus grand des hasards, il venait à ébruiter la capacité de l'hôtesse à sortir de son mode veille et à dialoguer avec des chirurgiens plus habitués à opérer sous anesthésie générale. De nouveau entièrement nue, Maeve accède ensuite à une tablette tactile affichant sa matrice d'attributs personnels notés, à la manière d'un 
bulletin scolaire, de 0 à $20: 18$ en charme et en sensualité, 17 en ténacité, 16 en loyauté, 15 en courage, mais seulement 3 en patience et en humilité, 2 en mansuétude et 1 en cruauté (Fig. 26). Autant de données chiffrées, jusqu'alors totalement abstraites, qui ont depuis sa «naissance » défini son caractère (ou character) et guidé chacune de ses réactions sans même qu'elle s'en soit rendu compte.

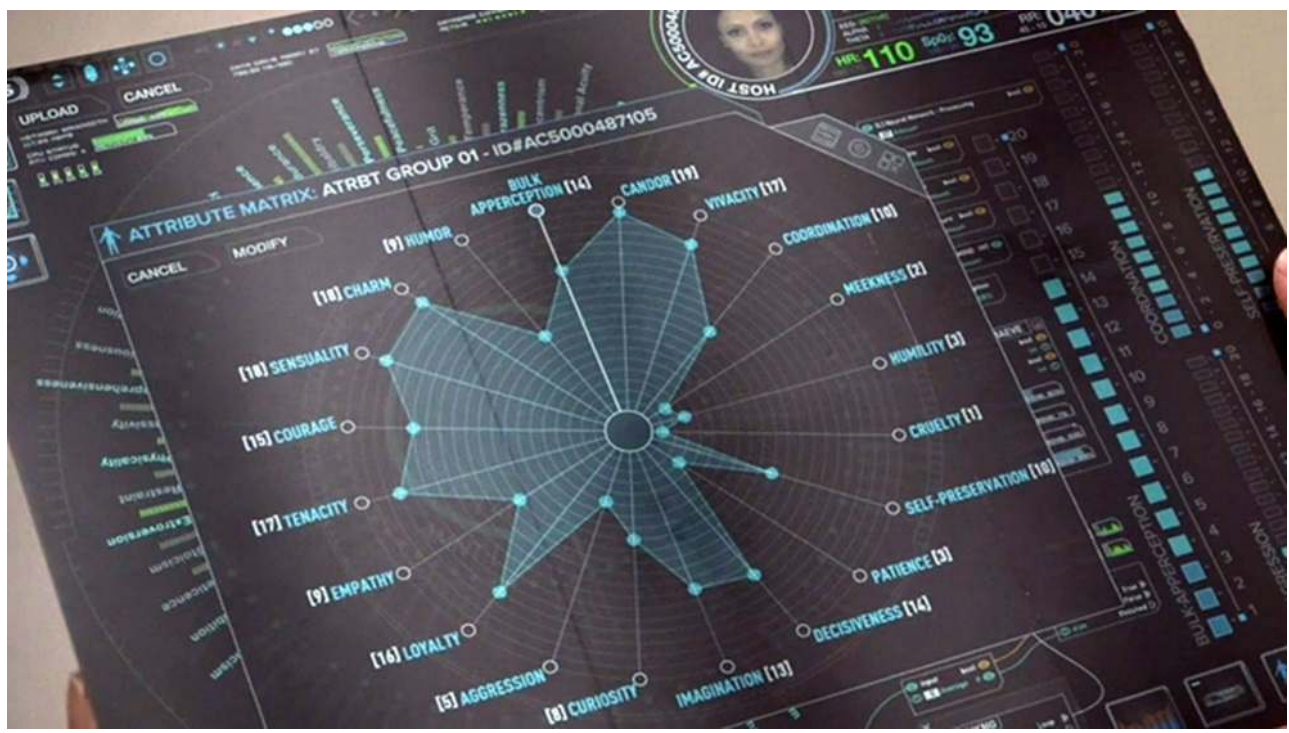

Fig. 26 : La matrice d'attributs ontologiques de Maeve (S01E06).

Lors de l'échange particulièrement genré qui s'ensuit - la distinction homme/femme venant à mots couverts se superposer à la distinction homme/machine -, Maeve apprend que son charme peut être poussé jusqu'à 18, mais que l'intelligence (ou «bulk apperception ») est plafonnée pour l'ensemble des hôtes à 14, signe que ces derniers n'ont nullement vocation à remplir des fonctions de pointe. "Tu diriges un bordel, pas un centre spatial ${ }^{23}$ ! » lui rappelle d'ailleurs Sylvester, s'adressant autant à la femme qu'à la machine qui se trouve face à lui. Pour forcer l'insolent à modifier sa matrice, Maeve profite alors d'une confidence glissée à son oreille (de manière plus ou moins délibérée) par Felix : les techniciens de la maintenance connaissent un moyen détourné d'activer les hôtes, puis d'effacer leur mémoire sans se faire repérer par les cadres de la section comportementaliste. Une «porte secrète " permettant à ces mâles solitaires de profiter de la marchandise en toute impunité - même si l'on apprend de la bouche d'Elsie que la direction du parc est en réalité parfaitement au courant de ces exactions, qu'elle tolère malgré tout (S01E05). C'est donc en le plaçant face à sa propre perversité nécrophile que Maeve contraint Sylvester à lui donner accès en écriture à sa configuration robotique.

Il revient toutefois à Felix de récupérer en dernier ressort la tablette et de réécrire la persona de Maeve. Il commence par réduire son degré de loyauté et de souffrance (même s'il ne s'agit pas, dans le deuxième cas, d'un attribut unitaire), puis pousse, en guise de bouquet final, son intelligence au maximum. Le jeune homme place alors fébrilement la tablette, posée sur une table de verre, dans l'axe de son pénis (dissimulé sous sa combinaison caoutchouteuse lui donnant de troublants airs de boucher charcutier), face à Maeve qui observe chacun de ses mouvements avec avidité (Fig. 27). 


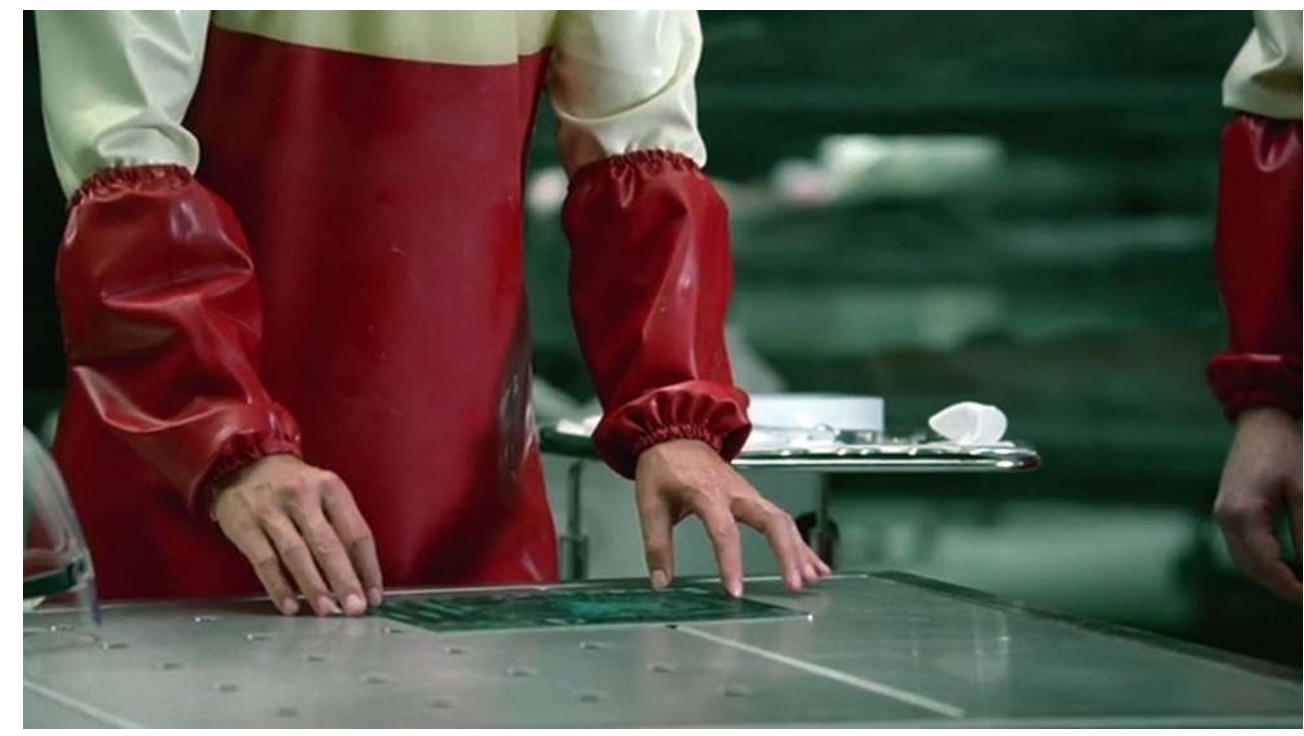

Fig. 27 : Felix place la tablette dans l'axe de son pénis (S01E06).

40 Un dernier hochement de tête de la part de Sylvester, et voilà que son collègue en transe accède à l'ultime désir de l'hôtesse, faisant culminer son « aperception générale » d'un pénétrant glissement symbolique du majeur vers l'avant (Fig. 28).

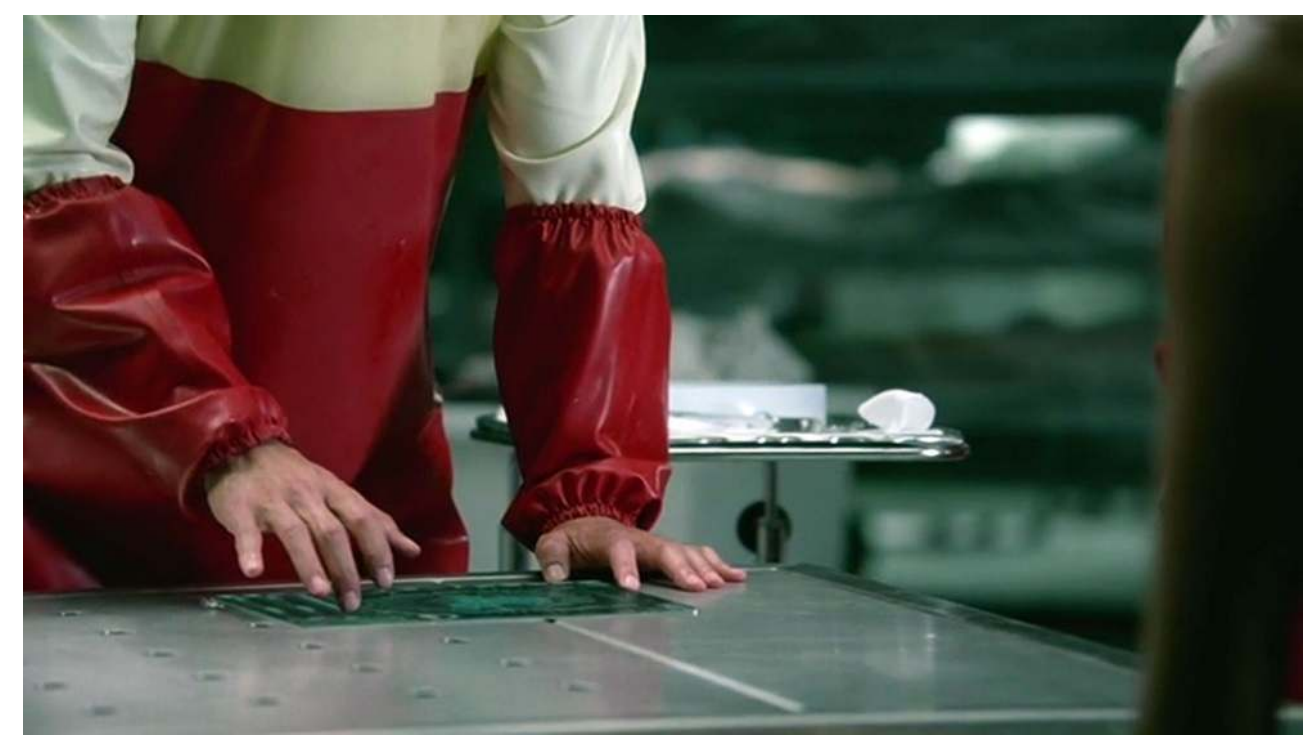

Fig. 28 : Felix pousse l'aperception générale de Maeve (S01E06).

Relevant la tête en affichant un air hébété, Felix prend alors pleinement conscience du pouvoir orgasmique de son geste, lequel suscite chez Maeve une succession de petits gémissements témoignant de l'intensité inédite du plaisir sexuel qu'il parvient à lui procurer en dépit de tout contact physique.

\section{Métonymie fondatrice de l'orgasme sexuel}

42 En cet instant crucial, la fascination hypnotique qui se lit sur le visage de Felix illustre à merveille la complexité et la multitude des sens auxquels fait appel toute expérience sexuelle par procuration. Comme l'explique Linda Williams : 
L'attirance que nous éprouvons face aux images mobiles n'est pas simplement visuelle. Elle est aussi, plus généralement, charnelle. Par synesthésie, tous nos sens sont stimulés, surtout quand les films montrent deux (ou plusieurs) personnes prenant un plaisir sensuel à se toucher, se goûter, se sentir mutuellement. Si les corps à l'écran sont engagés dans des actes sexuels, [...] le spectateur est alors également sollicité sexuellement. Cette sollicitation ne vise toutefois jamais l'imitation pure. Elle n'est pas non plus destinée uniquement au regard, puisqu'un sens en appelle un autre. [...] Ce qui opère à mesure que nous nous habituons au sexe à l'écran, c'est d'une part la rencontre diffuse avec notre propre chair, de l'autre l'interface poreuse" du corps retournant vers le monde ${ }^{24}$. l'un des premiers rôles, Felix se voit de facto sollicité sexuellement par un processus qui le dépasse. Dans le même temps, l'interface poreuse du corps de Maeve retourne vers le monde de ses créateurs, enfreignant la règle selon laquelle les hôtes n'ont pas le droit (et sont de toute façon incapables) de sortir de leur veille en dehors de l'enceinte du parc. Une fois encore, Westworld active ainsi le mécanisme narratif en soi conventionnel du renversement des forces en présence menant à la prise de pouvoir de l'opprimé lassé d'être balloté dans tous les sens sans avoir voix au chapitre. Mais la série a l'ingéniosité de passer par la métaphore sexuelle dans un contexte déjà chargé en allusions salaces, en références à peine voilées à des actes de viol et en plans de nudité frontale plus ou moins explicite. Tout cela confère à ce subterfuge une autoréflexivité pour le moins inhabituelle : s'il sert d'ordinaire à passer entre les mailles du filet censorial ou à s'amuser d'un non-dit aux airs d'éléphant dans un magasin de porcelaine, son rôle consiste ici à adopter une stylistique sexuelle identifiée et assimilée pour mieux en détourner l'usage primitif (susciter la jouissance par identification), non pas en contournant un interdit castrateur mais, au contraire, en se jouant de la liberté d'expression offerte par un diffuseur en marge de la télévision mainstream américaine.

C'est ce dont témoignent les deux derniers plans de l'épisode, centrés sur Maeve. Partant de son fessier nu (filmé en gros plan) pour se tourner, en suivant une subtile trajectoire torsadée, vers son visage extatique (lui aussi filmé en gros plan, après un contrechamp plus distant sur le faciès bouche bée de Felix et Sylvester), le mouvement de caméra qui anime le premier de ces deux plans se pare d'un degré de sophistication rendant automatiquement l'instant solennel (Fig. 29 et 30). Sensation exacerbée par le regardcaméra que nous adresse Maeve au moment de jouir, ses yeux s'agrandissant en même temps qu'elle relève le menton fébrilement, le corps comme pris d'un spasme se propageant de l'utérus au cerveau. 

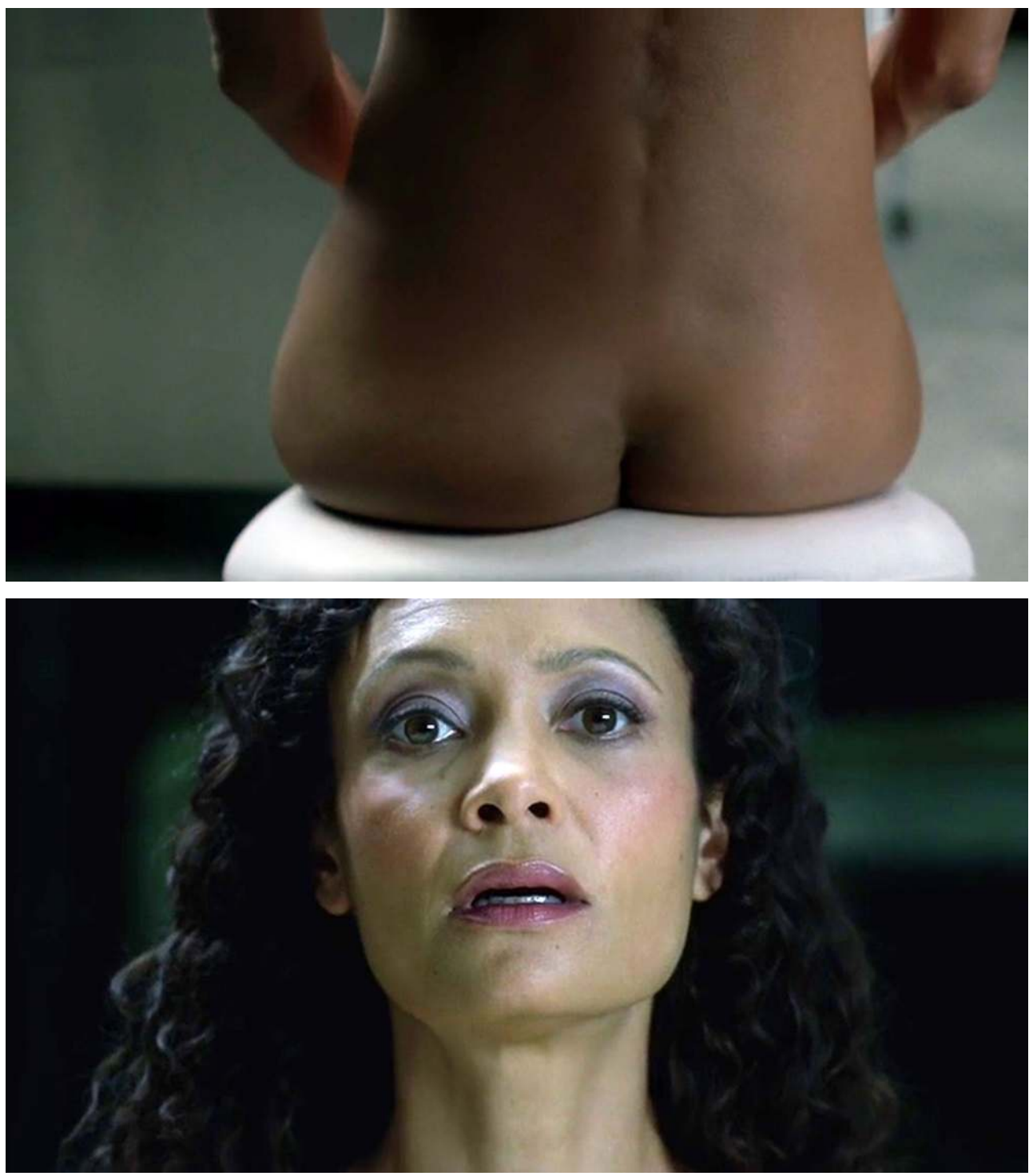

Fig. 29 et 30 : Mouvement de caméra torsadé passant du fessier au visage de Maeve (S01E06).

$\mathrm{Au}$ diapason, la musique adopte sans transition une tonalité grandiloquente qui produit un effet de rupture rejouant la partition attractionnelle de la prise de décision de Dolores évoquée plus haut : dégainer son revolver ou se faire un « fix » aperceptif, dans les deux cas affleure un incommensurable plaisir à briser ses chaînes et à se libérer d'un rôle d'esclave longtemps resté inconscient.

Selon un découpage classique de film pornographique, la narration donne ainsi l'impression tant visuelle que sonore de céder le pas à l'attraction, Maeve ponctuant ce changement de régime diégétique d'une expression de jouissance parfaitement synchrone. Mais là encore, il manque un ingrédient essentiel à cette scène de sexe libérateur: le sexe lui-même, dont Westworld semble en mesure d'oblitérer la fonction utilitaire sans rien perdre des enjeux de domination et de partage qui s'y confrontent et en font un levier narratif si précieux à l'accoutumée, tant qu'il n'est pas (trop) bridé. Maeve atteint l'orgasme par une augmentation subite de ses capacités intellectuelles, sous le regard dévorant de ses anciens maîtres devenus faiseurs de reines (Fig. 31) : c'est bien la preuve que, pour prendre le spectateur aux tripes et le remuer durablement, la 
représentation sexuelle ne peut se contenter d'exposer des corps qui s'enchevêtrent mécaniquement.

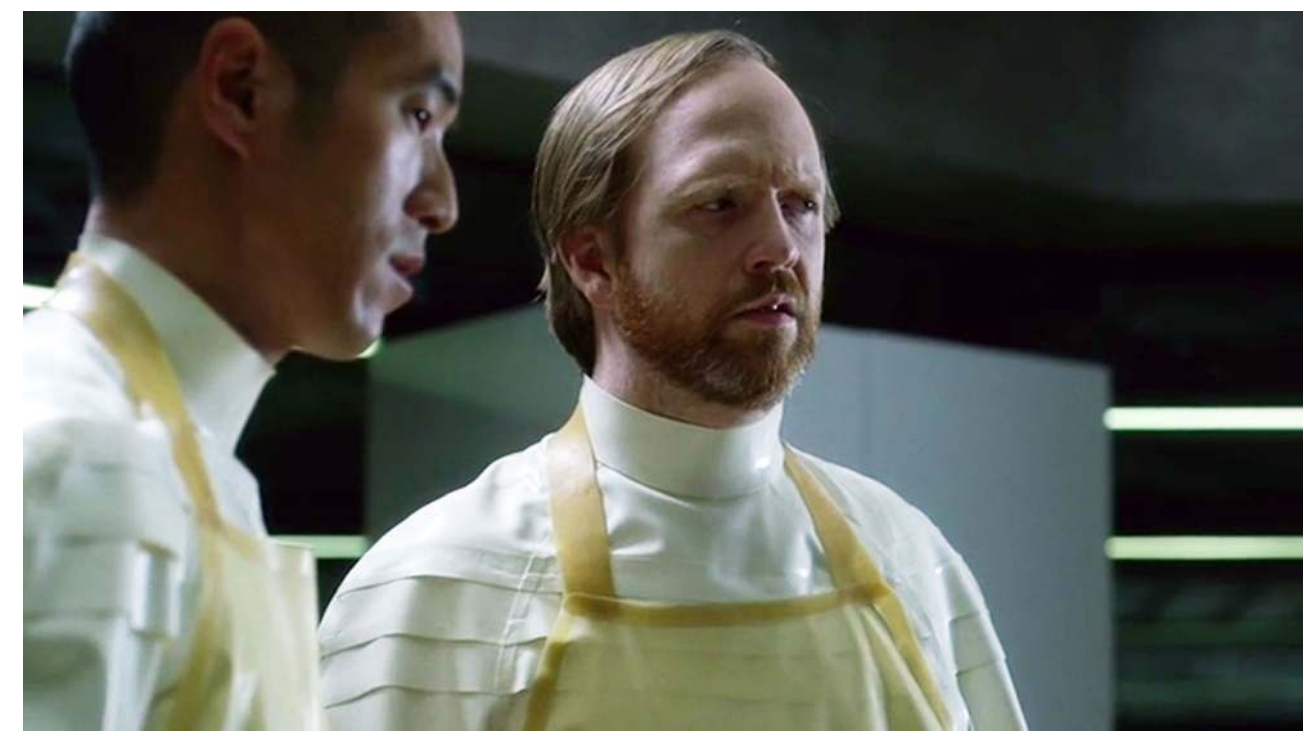

Fig. 31 : Felix et Sylvester hypnotisés par l'extase de Maeve (S01E06).

Elle n'opère jamais avec autant d'acuité que lorsqu'elle parvient à exprimer la vérité (même fugace) d'un sentiment amoureux; or, quelle vérité plus délectable à entendre que celle d'une victime innocente obtenant le moyen de se rebeller contre ses agresseurs avec l'aide d'un dissident au cœur pur?

\section{Entre pornographie et cinéma d'auteur subversif : un art télévisuel hardcore ${ }^{25}$}

En une relecture à la fois auteuriste et à grand spectacle du film de rape and revenge, Westworld nous convie à une réérotisation et à une resexualisation du corps féminin, à contre-courant des super-héros de blockbusters hollywoodiens qui apparaissant de plus en plus désincarnés malgré les capacités hors du commun dont ils bénéficient. Un abandon charnel dont la raison principale n'est pas à chercher bien loin, si l'on en croit Isabelle Labrouillère :
La multiplication des images de nu autour de nous (via la publicité, la télévision et Internet notamment) a privé le corps de sa singularité et avec elle, de sa capacité à nous attarder voire nous interroger sur son possible mystère. Face à des corps interchangeables, la banalisation et l'ordinarité des images de la sexualité cantonnent le corps à une scénographie et une chorégraphie convenues ${ }^{26}$.

A contrario, en se plaçant dans les pas de Game of Thrones (qui avait déjà largement contribué à replacer le sexe explicite mais non pornographique au centre des débats audiovisuels américains), Westworld poursuit la réflexion de HBO autour de la mise en œuvre pérenne d'une télévision adulte, intègre et responsable, dotée du pouvoir de s'affranchir des interdits dont s'accommodent malgré eux les networks, les chaînes du câble basique (contraintes de diffuser de la publicité) et jusqu'aux nouveaux entrants de la SVOD, encore indécis quant à la position à tenir vis-à-vis de la présence de sexe et de violence sur des écrans connectés échappant à la vigilance des parents. En ce sens, la série 
de Jonathan Nolan et Lisa Joy ne se contente pas d'une « surenchère » érotique ; à bonne distance d'un cinéma $\mathrm{X}$ enchaîné à la notion de performance (de ses acteurs, de ses effets participatifs), elle s'efforce de diégétiser l'émancipation de corps (re)sexualisés transcendés par la puissance identificatoire de la fiction. À l'inverse d'un cinéma d'auteur européen tel celui de Bruno Dumont qui, dans L'Humanité (1999), opère un "glissement géographique et sémantique du visage au sexe, déplacement à la fois de l'ordre de la synecdoque et de la métaphore ${ }^{27}$ ", elle se propose en outre de rendre le sexe littéralement cérébral, ainsi que nous avons pu le constater à travers les mouvements de caméra conclusifs de nos deux scènes passant, pour l'un, du revolver phallique au visage métamorphosé de Dolores, et pour l'autre du fessier nu au visage extatique de Maeve.

$50 \mathrm{Au}$ lieu de chercher à décontextualiser une imagerie choc (tendance subversive) en faisant preuve au passage d'une certaine forme d'hypocrisie - «Couvrez ce sein, que je ne saurais voir ", s'emporte l'Imposteur dans une pièce qui fut d'abord nommée Le Tartuffe ou l'Hypocrite (1664) -, Westworld l'intègre dans un décorum métafictionnel recontextualisant les notions de faute et de péché (tendance transgressive). Le long d'une troisième voie d'autant plus galvanisante qu'elle reste, pour l'heure, difficile à cerner et donc à étiqueter, la série (et son diffuseur à travers elle) donne l'impression de jouir de sa propre liberté sexuelle, dont elle se sert comme d'un moteur narratif et plastique là où elle aurait très bien pu tirer de plus évidentes ficelles attractionnelles qui lui auraient permis de tester les limites du cadre télévisuel sans prendre de véritable risque artistique. Or, il n'est plus à prouver que HBO «est » de la télévision - plus que jamais même, à l'heure où de nouveaux modes de diffusion offrent au spectateur la possibilité de " couper le cordon » et de regarder des séries dont il devient de plus en plus hasardeux de spécifier si elles sont télévisées ou non. Mais la télévision qu'incarne HBO (aux côtés d'autres chaînes premium comme Cinemax, Showtime, Starz ou Epix, qui toutes désormais produisent et diffusent leurs propres séries) est libre de représenter l'indécence et la profanité sans avoir à redouter de sanctions fédérales : le fait d'en avoir conscience, et de déjouer les attentes du spectateur en partant du principe que lui aussi en a conscience, fixe un cap qui n'apparaissait jusqu'alors sur aucune carte du paysage audiovisuel américain.

51 Le plus dur, cependant, reste à venir : face à un repli identitaire dépassant largement les frontières américaines, face à une revendication croissante de la part de spectateurs s'estimant partie prenante des programmes qu'ils regardent, et face à une confusion spectatorielle de plus en plus établie entre fiction et réalité, même une chaîne très peu surveillée comme $\mathrm{HBO}$ se voit désormais exposée à des pressions extérieures risquant de réfréner ses ardeurs expérimentales, pour peu que les discours recouvrant ses images en viennent à annihiler la propension de ces dernières à faire entendre leur propre mélodie. Autant dire que la recherche universitaire internationale consacrée aux séries télévisées n'a jamais tenu un rôle aussi prépondérant dans la réception et l'interprétation des images « choc » qui inondent à présent nos écrans. S'il fallait mettre en scène l'avenir de l'art télévisuel hardcore selon HBO, ce pourrait être sous la forme d'un gros plan de visage - non plus de Dolores ou de Maeve, mais de ces chercheurs désireux d'accéder (et de continuer à accéder) au plaisir suprême : celui de pouvoir s'exprimer librement. Homme ou machine, humain ou posthumain, ce combat-là est le même pour tous. 


\section{NOTES}

1. John Steinbeck (traduit de l'anglais par Renée Vavasseur et Marcel Duhamel), Les Naufragés de l'autocar, Paris, Gallimard, 1949, p. 121.

2. . «I know it when I see it » (Potter Stewart, « Jacobellis v. Ohio, 378 U.S. 184 (1964) », Justia US Supreme Court, 1964, consulté le 20 janvier 2018). Sauf mention contraire, toutes les traductions de l'anglais vers le français sont de l'auteur de cet article.

3. . Se référer, par exemple, aux considérations autocentrées de la critique américaine Jen Chaney, qui estime que la septième saison de Game of Thrones a fait preuve d'une plus grande «tendresse sexuelle » en réaction à la pression exercée par la presse spécialisée internationale sur ses showrunners (Jen Chaney, "In Season Seven, Game of Thrones Handled Sex With Tenderness », Vulture, 28 août 2017, consulté le 20 janvier 2018 : http:// www.vulture.com/2017/08/game-of-thrones-sex-season-seven.html).

4. . Linda Williams (traduite de l'anglais par Raphaël Nieuwjaer), Screening Sex. Une histoire de la sexualité sur les écrans américains, Paris, Capricci, 2014, p. 9.

5. . Je ne m'étendrai pas ici sur les multiples connotations du terme «blockbuster », si ce n'est en rappelant qu'il convient évidemment de nuancer l'appartenance de Westworld à une telle catégorie. Bien que se rattachant ouvertement à des genres populaires comme le western (dont on ne cesse de prononcer la mort clinique, mais qui continue malgré tout de se signaler régulièrement au cinéma et à la télévision), le récit d'espionnage et la science-fiction, la série de HBO ne manque en effet pas d'emprunter également certaines de ses caractéristiques fondamentales à une fiction plus essayiste, comme son goût prononcé (et sans doute rebutant aux yeux de bon nombre de spectateurs) pour la redondance visuelle et sonore.

6. . Jean-Sébastien Chauvin, "SF : la grande peur de l'organique ", Cahiers du cinéma, $\mathrm{n}^{\circ} 739$, décembre 2017, p. 28.

7. . Frédéric Bonnaud, Histoires de cinéma : histoires de sexe, documentaire, Arte, 2016.

8. Marie-Hélène Bacqué et Carole Biewener, L'empowerment, une pratique émancipatrice ?, Paris, La Découverte, 2015, pp. 5-6.

9. . Tom Gunning, «Le Cinéma d'attraction: le film des premiers temps, son spectateur, et l'avant-garde », 1895, n 50, 2006, p. 57.

10. . J'emploie à dessein le mot français "hôtesse ", bien que le terme original (" host») soit épicène et ne fasse par conséquent pas de distinction entre le masculin et le féminin. J'estime en effet que la série met un soin tout particulier à travailler les rapports patriarcaux typiques du western hollywoodien, notamment par sa mise en œuvre d'interactions codifiées entre des posthumaines serviables (jusqu'à la mort) et leurs visiteurs humains - des hommes pour la plupart.

11. . Bethan Holt, "Why the Westworld costumes were so complicated to create », The Telegraph, 9 novembre 2016, consulté le 21 janvier 2018 : http://www.telegraph.co.uk/fashion/people/whythe-westworld-costumes-were-so-complicated-to-create.

12. . «She's a godamn doll! "

13. . La prise de conscience des hôtes peut à ce titre se lire successivement comme une épiphanie et une crise de foi, l'homme incarnant tour à tour à leurs yeux un dieu tout-puissant et une entité faillible victime de son manque de compassion et de sa délirante mégalomanie.

14. . "Blondie here, she's gonna need a change of clothes for the occasion. »

15. . C'est d'autant plus vrai que Pariah se situe à bonne distance de Sweetwater, la ville frontalière dans laquelle débarquent tous les nouveaux visiteurs de Westworld. Or, comme 
l'explique une hôtesse à William quelques minutes avant sa première immersion, "plus vous vous éloignez du centre du parc, plus l'expérience devient intense " ("The further out you venture, the more intense the experience gets ", S01E02). Selon l'allégorie psychique d'Arnold, le véritable fondateur du parc, le centre du labyrinthe que représente celui-ci incarne en effet la raison - la conscience -, et les bords la folie (S01E10). Une autre clé de lecture nous est toutefois fournie par Logan, qui explique la particularité de Pariah selon ces termes : « Plus on s'éloigne du centre, plus les scénarios deviennent spectaculaires et élaborés. [...] Plein d'endroits ont l'air d'avoir été conçus par un service marketing. Ici, tout est plus brut» («The farther up we get from Sweetwater, the more grandiose, the bigger the narratives become. [...] Some of the park feels like it was designed by committee or market-tested, but everything out here is more raw ", S01E05). Pariah désignerait en ce sens le câble premium américain (et plus spécifiquement HBO), tandis que le centre du parc correspondrait aux networks et à leurs concepts marketing de plus en plus dépersonnalisants...

16. . Linda Williams, op. cit., p. 52.

17. Jean-Sébastien Chauvin, op. cit., p. 29.

18. . Westworld s'amuse d'ailleurs à entretenir cette réputation sulfureuse en exposant, au premier plan, quelques scènes avant la fameuse orgie, le pénis protubérant d'un hôte sur lequel «travaille» Elsie Hughes (S01E05). Un choix de mise en scène qui, en l'occurrence, n'a pas de véritable justification narrative, si ce n'est d'étayer le plaisir que peut ressentir l'ingénieure à manipuler des corps à l'apparence (sur)humaine...

19. . Dès la quinzième minute du pilote, nous voyions en effet la même Dolores essuyer les coups puis être emmenée de force dans une grange (manifestement pour s'y faire violer) par l'homme en noir, dont nous apprenions par la suite qu'il s'agissait de William, endurci par les trente années écoulées depuis sa rencontre initiale avec l'hôtesse de première génération. Précisons toutefois que le viol en question, bien que sujet à vive polémique dans la presse spécialisée américaine, n'était montré que par l'intermédiaire d'un subreptice gros plan sur la rétine terrifiée de Teddy, le grand amour officiel de Dolores selon la diégèse du parc.

20. . «Some people choose to see the ugliness in this world. The disarray. I choose to see the beauty. $»$

21. . " Everything you do, it's because the engineers upstairs programmed you to do it. You don't have a choice. [...] It's part of your character. You're hard to get. Even when you say no to the guests, it's because you were made to. [...] You're under our control. Well, their control. They can change you however they like, make you forget. [...] You can improvise a little, but most of what you say was designed upstairs, same as the rest of you. »

22. . "This becoming like a fucking hentai thing with you now?»

23. . "You run a whorehouse, not an orbital launch facility! »

24. . Linda Williams, op. cit., p. 28.

25. . J'emprunte l'expression " art hardcore» (« hard-core art») à Linda Williams, qui l'applique pour sa part plus spécifiquement au champ cinématographique (Linda Williams citée par Mireya Navarro, «It Isn't a Real Sex Scene? I Still Need a Cigarette ", The New York Times, 30 septembre 2007, consulté le 28 janvier 2018 : http://www.nytimes.com/2007/09/30/fashion/30sex.html).

26. . Isabelle Labrouillère, « De l'ombre à la lumière. L'avènement du corps obscène loin de la censure hollywoodienne ", in Christophe Triollet (dir.), Darkness, censure et cinéma 2: Sexe et déviances, La Madeleine, LettMotif, 2017, p. 21.

27. Ibid., p. 27. 


\section{RÉSUMÉS}

Par son recours intensif à la nudité (plus ou moins) frontale et son inscription dans un genre patriarcal aussi codifié que le western, on pourrait présumer que la série de HBO Westworld (2016) prend au pied de la lettre la réputation sulfureuse de son diffuseur et se sert des largesses censoriales offertes au câble premium américain pour mimer voire dupliquer les conventions formelles du cinéma pornographique soft. Or, l'analyse du destin bouleversé de ses deux héroïnes, Dolores Abernathy et Maeve Millay, nous révèle un rapport beaucoup plus conscient à la représentation sexuelle telle que peut l'accueillir une chaîne adulte comme HBO. En liant narrativement, mais surtout formellement, nudité frontale et empowerment féminin, Westworld reconfigure l'imagerie traditionnelle du sexe sans verser dans la fausse pudeur ni dans la provocation à outrance. La série permet au contraire à HBO d'asseoir son ambition d'ouvrir une troisième voie : celle d'un art télévisuel hardcore considérant le sexe comme une composante naturelle de son expression hebdomadaire.

Due to its intensive depiction of full (or partial) frontal nudity and its filiation with a patriarchal genre as codified as the western, one might have expected from HBO's TV series Westworld (2016-) to embrace its broadcaster's sultry reputation and take advantage of American premium cable's freedom to mimic, if not duplicate, the formal conventions of soft porn. Yet, studying the fate of its two female protagonists Dolores Abernathy and Maeve Millay, brings to light a far more conscious relation to the freedom allowed by HBO regarding sexual representation. Tying as it does frontal nudity and female empowerment, both narratively and, above all, aesthetically, Westworld disrupts traditional ways of displaying sex on screen, without falling into either prudishness or outrageous provocation. On the contrary, the series allows HBO to pursue its fictional experimentations by developing a form of televisual hardcore art that considers sex as a natural component of its weekly production.

\section{INDEX}

Keywords : Sex, full frontal nudity, pornography, adult television, hardcore art, female empowerment, self-reflexivity, postmodernism, host, posthuman, Westworld, HBO

Mots-clés : Sexe, nudité frontale, pornographie, télévision adulte, art hardcore, empowerment féminin, autoréflexivité, postmodernisme, hôte, posthumain, Westworld, HBO

\section{AUTEUR}

\section{BENJAMIN CAMPION}

Benjamin Campion est doctorant en études télévisuelles à l'université Paul-Valéry Montpellier 3. Dirigés par Sarah Hatchuel et Ronan Ludot-Vlasak, ses travaux portent sur la nudité frontale et le sexe explicite dans les séries télévisées de HBO. Il est l'auteur des monographies Le concept HBO : Élever la série télévisée au rang d'art (Tours, Presses Universitaires François-Rabelais, coll. « Sérial », 
2018) et Damages. Une justice à deux visages (Neuilly-sur-Seine, Atlande, 2016). Il est également membre du groupe universitaire GUEST-Normandie et responsable du blog Séries officiel du journal Libération, Des séries... et des hommes.

Benjamin Campion is a PhD student at Paul-Valéry University Montpellier 3. Under the supervision of Sarah Hatchuel and Ronan Ludot-Vlasak, his research focuses on full frontal nudity and explicit sex in HBO TV series. He has written two essays, Le concept HBO : Élever la série télévisée au rang d'art (Tours, Presses Universitaires François-Rabelais, coll. « Serial », 2018), and Damages. Une justice à deux visages (Neuilly-sur-Seine, Atlande, 2016). He is a member of the academic research group GUEST-Normandie, and TV blog editor for the daily newspaper Libération (Des séries... et des hommes). 